\title{
Autonomous Vehicle Maneuvering at the Limit of Friction
}

\section{Victor Fors}

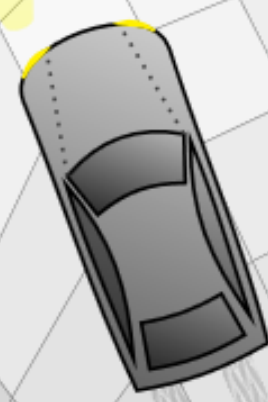





\title{
Autonomous Vehicle Maneuvering at the Limit of Friction
}

\author{
Victor Fors
}

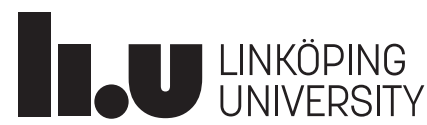

Division of Vehicular Systems

Department of Electrical Engineering

Linköping University

SE-581 83 Linköping, Sweden

Linköping 2020 
Cover picture: The cover page picture is an illustrated version of a force-slip diagram showing the tire slips and forces visited during a maneuver. This is sometimes called a Nielsen diagram.

Copyright () 2020 Victor Fors, unless otherwise noted ISBN 978-91-7929-770-1

ISSN 0345-7524

URL http://urn.kb.se/resolve?urn=urn:nbn:se:liu:diva-170606

Published articles have been reprinted with permission from the respective copyright holder.

Typeset using $\mathrm{LTT}_{\mathrm{E}} \mathrm{X}$

Printed by LiU-Tryck, Linköping, Sweden 2020 
To Sally 


\section{Populärvetenskaplig sammanfattning}

Självkörande fordon testas idag i olika pilotprojekt världen över. Ett självkörande fordon kan inte förlita sig på att det finns en förare som är uppmärksam och snabbt kan ta över, utan det måste själv kunna ta sig an alla situationer som kan uppstå. Med sikte på framtida säkerhetssystem som kan utföra undanmanövrar i utmanande situationer, studeras här autonoma fordonsmanövrar där däckens friktion mot marken utnyttjas till fullo. Dessa säkerhetssystem kan rädda många liv med sin potential att köra bättre än en professionell förare.

I den första delen av avhandlingen studeras hur ett fordon på bästa möjliga sätt kan hantera kritiska situationer som en undanmanöver. Genom att de optimala manövrarna räknas fram av optimeringsalgoritmer på en dator, kan man identifiera beteenden som kan ligga till grund för utformningen av nya säkerhetssystem. När optimal bromsning jämförs med hur det hade gått att bromsa annorlunda, visar det sig att däcken ska bromsas så att kraften blir den största möjliga i en given riktning.

Ett fordon som utnyttjar alla tillgängliga krafter mot marken är begränsad av friktionen mellan däck och mark. Genom att förenklat beskriva fordonet som en partikel med begränsad acceleration, kan man skatta i vilken riktning fordonets acceleration ska maximeras för att minimera risken för en kollision. Man behöver sedan bestämma hur fordonet ska styras och bromsas för att maximera accelerationen i denna riktning. För att göra detta utan alltför detaljerad kunskap om däckens egenskaper, utnyttjas bland annat att däcken i en kritisk manöver framförs på friktionsgränsen. Genom att manövrera enligt den minsta friktion som krävs för att kollision överhuvudtaget ska kunna undvikas, så kan man genomföra autonoma undanmanövrar utan kunskap om vägens faktiska friktion.

Vid ett autonomt filbyte som genomförs på minsta möjliga tid så visar det sig otillräckligt att modellera bilens accelerationsbegränsning vid skattning av önskad accelerationsriktning, utan det kräver även att man tar hänsyn till bilens tröghet att rotera. Detta går att hantera genom att man i modellen begränsar hur snabbt accelerationen kan förändras. Kan man inte analytiskt bestämma önskad accelerationsriktning, inte ens med en förenklad modell, så kan man låta en optimeringsalgoritm göra det åt en. En sådan lösning tillåter en avvägning mellan styrning och bromsning som minimerar risken för kollision i utmanande situationer. 


\section{Abstract}

Without a driver to fall back on, a fully self-driving car needs to be able to handle any situation it can encounter. With the perspective of future safety systems, this research studies autonomous maneuvering at the tireroad friction limit. In these situations, the dynamics is highly nonlinear, and the tire-road parameters are uncertain.

To gain insights into the optimal behavior of autonomous safetycritical maneuvers, they are analyzed using optimal control. Since analytical solutions of the studied optimal control problems are intractable, they are solved numerically. An optimization formulation reveals how the optimal behavior is influenced by the total amount of braking. By studying how the optimal trajectory relates to the attainable forces throughout a maneuver, it is found that maximizing the force in a certain direction is important. This is like the analytical solutions obtained for frictionlimited particle models in earlier research, and it is shown to result in vehicle behavior close to the optimal also for a more complex model.

Based on the insights gained from the optimal behavior, controllers for autonomous safety maneuvers are developed. These controllers are based on using acceleration-vector references obtained from friction-limited particle models. Exploiting that the individual tire forces tend to be close to their friction limits, the desired tire slip angles are determined for a given acceleration-vector reference. This results in controllers capable of operating at the limit of friction at a low computational cost and reduces the number of vehicle parameters used. For straight-line braking, ABS can intervene to reduce the braking distance without prior information about the road friction. Inspired by this, a controller that uses the available actuation according to the least friction necessary to avoid a collision is developed, resulting in autonomous collision avoidance without any estimation of the tire-road friction.

Investigating time-optimal lane changes, it is found that a simple friction-limited particle model is insufficient to determine the desired acceleration vector, but including a jerk limit to account for the yaw dynamics is sufficient. To enable a tradeoff between braking and avoidance with a more general obstacle representation, the acceleration-vector reference is computed in a receding-horizon framework.

The controllers developed in this thesis show great promise with low computational cost and performance not far from that obtained offline by using numerical optimization when evaluated in high-fidelity simulation. 


\section{Acknowledgments}

At my places of employment I have been lucky to have great coworkers, and the Vehicular Systems group at Linköping University where this research has been carried out is no exception. I would like to thank my past and present coworkers for a supportive work environment and productive coffee breaks. I would specifically like to thank Lars Nielsen and Björn Olofsson for their enduring role as coauthors, taking the time to be involved in our papers. I am of course also grateful to my other great coauthors Yangyan Gao, Tim Gordon, and Pavel Anistratov for joining forces with us. Additionally, I would like to thank Lars Nielsen, Björn Olofsson, and Jan Åslund for their roles as supervisor and cosupervisors.

Other people I want to thank are Maria Hamnér and Vaheed Nezhadali for showing me the way around the office when I was new at Vehicular Systems, Kristoffer Lundahl and Pavel Anistratov for being office mates that I can disturb, my father for being proud, my mother for being worried, my sister for being supportive, Sally for being here, and Lars, Björn and Pavel again for really helping out with the final stretch to complete this thesis, and any other who influenced its quality.

Linköping, October 2020

Victor Fors

\section{Funding}

The author is a member of ELLIIT, the Strategic Area for ICT research, supported by the Swedish Government. This work was partially supported by the Wallenberg AI, Autonomous Systems and Software Program (WASP) funded by the Knut and Alice Wallenberg Foundation. 


\section{Contents}

1 Introduction 1

1.1 Background . . . . . . . . . . . . . . . 1

1.2 Motivation . . . . . . . . . . . . . . . . . . . 4

1.3 Outline ..................... 5

2 Vehicle Modeling $\quad 7$

2.1 World Coordinate Systems . . . . . . . . . . . . . . . . 7

2.2 Chassis Models . . . . . . . . . . . . . . . . . 11

2.3 Wheel and Tire Modeling . . . . . . . . . . . . . . . 23

2.4 Discussion . . . . . . . . . . . . . . . . . . 31

3 Conclusion 35

3.1 Contributions . . . . . . . . . . . . . . . 35

3.2 Reflection . . . . . . . . . . . . . . . . . 42

3.3 Outlook ....................... . . 43

A Vehicle Model Parameters $\quad 45$

B The Modified Hamiltonian Algorithm 49

References 53

$\begin{array}{ll}\text { Papers } & 61\end{array}$ 
I Formulation and Interpretation of Optimal Braking and Steering Patterns

Towards Autonomous Safety-Critical Manoeuvres 63

1 Introduction . . . . . . . . . . . . . . . . . . . 64

2 Modelling ................. 66

3 Scenarios . . . . . . . . . . . . . . . 68

4 Optimal Control Problem Formulation . . . . . . . . . . . 71

5 Results ..................... . . 72

6 Discussion . . . . . . . . . . . . . . . 81

7 Conclusions . . . . . . . . . . . . . . 87

References .................. 88

II Attainable Force Volumes of Optimal

Autonomous At-the-Limit Vehicle Manoeuvres $\quad 91$

1 Introduction . . . . . . . . . . . . . . . . . 92

2 Scenarios ...................... 94

3 Modelling and Optimisation . . . . . . . . . . . . 97

4 Forces . . . . . . . . . . . . . . . . . . 100

5 Control Forces During the Left-Hand Turn . . . . . . . . . 108

6 Attainable Forces During the Left-Hand Turn . . . . . . . 110

7 Attainable Force Volumes During Critical Manoeuvres . . 111

8 Global Forces for Controller Design . . . . . . . . . . . 118

9 Conclusions . . . . . . . . . . . . . . . . . . 119

References . . . . . . . . . . . . . . . . 120

III Yaw-Moment Control At-the-Limit of Friction Using Individual Front-Wheel Steering and Four-Wheel Braking 125

1 Introduction . . . . . . . . . . . . . . . 126

2 Vehicle Model . . . . . . . . . . . . . . . . . 126

3 Modeling Combined Tire Forces At-the-Limit of Friction . 127

4 Chassis Control . . . . . . . . . . . . . . . . . . . . . 129

5 Results \& Discussion . . . . . . . . . . . . . . . . . . . 135

6 Conclusions . . . . . . . . . . . . . . . . . . . . . 142

References . . . . . . . . . . . . . . . . . . 142 
IV Autonomous Wary Collision Avoidance 145

1 Introduction . . . . . . . . . . . . . . . . 146

2 Overview of Controller Principles . . . . . . . . . . . 147

3 Center-of-mass acceleration reference . . . . . . . . . . 149

4 Tire Modeling for Real-Time Control . . . . . . . . . . . . 158

5 Online Avoidance Control . . . . . . . . . . . . . . . . . 162

6 Simulation Results . . . . . . . . . . . . . . . . . . . . 169

7 Discussion \& Conclusions . . . . . . . . . . . . . . 176

References . . . . . . . . . . . . . . . 177

V Real-Time Minimum-Time Lane Change

Using the Modified Hamiltonian Algorithm 181

1 Introduction . . . . . . . . . . . . . . . 182

2 The Modified Hamiltonian Algorithm . . . . . . . . . . . 182

3 Predicting the Switching Time . . . . . . . . . . . . . . 184

4 Stabilization Using MHA . . . . . . . . . . . . . . . 186

5 Results \& Discussion . . . . . . . . . . . . . . . . . . . . 187

6 Conclusions . . . . . . . . . . . . . . . . . 190

References . . . . . . . . . . . . . . . . . . . 191

VI Predictive Force-Centric Emergency Collision Avoidance by Steering and Individual Four-Wheel Braking 193

1 Introduction . . . . . . . . . . . . . . . . . . . . 194

2 Real-Time Motion Planning . . . . . . . . . . . . . 195

3 Acceleration References . . . . . . . . . . . . . . . . . . 197

4 Acceleration Following . . . . . . . . . . . . . . . 202

5 Scenarios . . . . . . . . . . . . . . . . . . 209

6 Evaluation Model . . . . . . . . . . . . . . . . . . 210

7 Results . . . . . . . . . . . . . . . . . . 213

8 Discussion . . . . . . . . . . . . . . . . . . . 222

9 Conclusions . . . . . . . . . . . . . . . . . . 224

References . . . . . . . . . . . . . . . . . 224 



\section{Chapter 1}

\section{Introduction}

/ ODERN passenger cars are not only defined by their hardware, but also by the millions of lines of code used to control and monitor their systems. Cars are traditionally controlled by human drivers, who have to rely on their senses and driving knowledge to handle any situation that may arise. Today, there are many pilot projects around the world with self-driving cars being tested on public roads. Without being able to fall back to a driver, a self-driving car needs to autonomously handle any situation it encounters. Although this is a monumental task, selfdriving cars have the potential to outperform the driving performance of even professional drivers. With the perspective of future high-performing autonomous safety systems, this thesis studies vehicle maneuvering at the limit of friction, where all the available tire-road friction is being utilized to mitigate danger. To this end, optimal behavior in safety-critical vehicle maneuvers is studied, and insights from this are used to design controllers that operate at the limit of the tire-road friction.

\subsection{Background}

Since the mid-20th century, the number of fatalities due to automotive accidents in Sweden have decreased in number even though the number of vehicles on the road have increased dramatically. In the year 1950, automotive accidents in Sweden resulted in 172.5 fatalities per 100,000 cars; the year 2018 that number was 5.8 fatalities per 100,000 cars [1]. Up until the 1960s, the focus in automotive safety was to improve the 


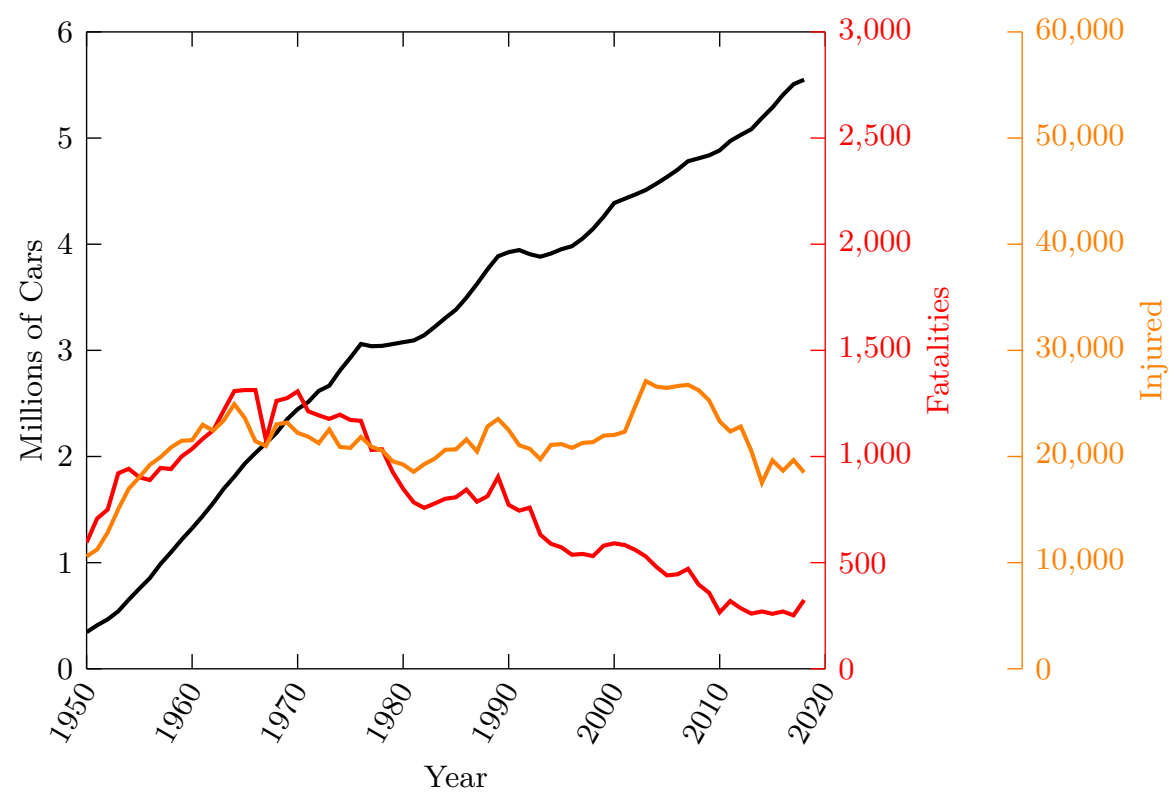

Figure 1.1: Number of cars, fatalities, and injured in traffic by year in Sweden between 1950 and 2018 [1].

road quality, but not until regulatory changes introduced speed limits on country roads, mandatory vehicle inspections, laws on seat belts, laws on minimum tire-thread depth, and more, did the increasing number of fatalities each year slow down to reach its peak in 1966-1967 [2]. Improvements of the vehicles themselves, such as designing the interior of a vehicle from a safety standpoint can drastically increase the chance of survival in the case of an automotive accident [3]. These safety improvements are referred to as passive safety systems, which include safety features such as seat belts, air bags, and deformation zones. However, passive safety systems do not prevent accidents from happening, but significantly reduce the risk of serious injury or death. This contributes to the fact that the number of injured in traffic are not decreasing as fast as the number of fatalities (see Figure 1.1).

Driver-assistance systems that can reduce the risk of accidents are referred to as active-safety systems. A commonly cited example is the anti-lock braking system (ABS), which prevents the loss of steering ability during heavy braking and can decrease the stopping distance [4]. Fitting a motorcycle with ABS reduces the risk of accidents and the risk of an 
accident being severe or fatal [5]. For cars, it is harder to prove that ABS reduces the risk of driver injury [6]. Specifically, ABS is not significantly reducing the risk of run-off-road crashes [7]. Thankfully, modern cars are also equipped with Electronic Stability Control (ESC), which reduces the risk of run-off-road crashes [8]. An ESC system uses differential braking to keep the vehicle response to steering input predictable for the driver [9], which is especially helpful in scenarios where the desired vehicle behavior is close to or beyond the capabilities of the vehicle.

Classic active-safety systems such as ABS and ESC rely on the driver to take in and process information about the surrounding vehicles and the road ahead, and provide appropriate input in terms of braking and steering. A driver-assistance system that adapts based on information about the surrounding vehicles is adaptive cruise control (ACC) [10]. ACC is an advancement of the classic cruise-control systems that in addition to keeping the vehicle driving at a reference velocity, also keeps the distance to the vehicle in front. ACC does still rely on being able to fall back on driver control and is not directly designed to prevent collisions. A corresponding function specifically designed for accident prevention is autonomous emergency braking (AEB), which applies heavy braking without any input from the driver to avoid collisions [11]. Cars with AEB have reduced risk for front-to-rear crashes and many injuries are estimated to be prevented as the function gains more widespread use [12].

More recently, systems for assisted driving have become available that can help with steering to keep the vehicle in its lane and more, but these still rely on an alert driver who pays attention and react promptly to challenging scenarios [13]. Even though these systems are getting more capable, they are not fully self-driving systems and require an engaged driver $[14,15]$. In an automated driving system as defined by SAE International [16] and adopted by the European Road Transport Research Advisory Council (ERTRAC) [17], the American agency National Highway Traffic Safety Administration (NHTSA) [18], and many others, the human driver is not required to monitor the driving environment and is at most only conditionally required to intervene. This definition means that these systems cannot rely on falling back on the driver when an unexpected safety-critical situation occurs, putting high requirements on their robustness, and creating a demand for autonomous solutions to critical situations. 


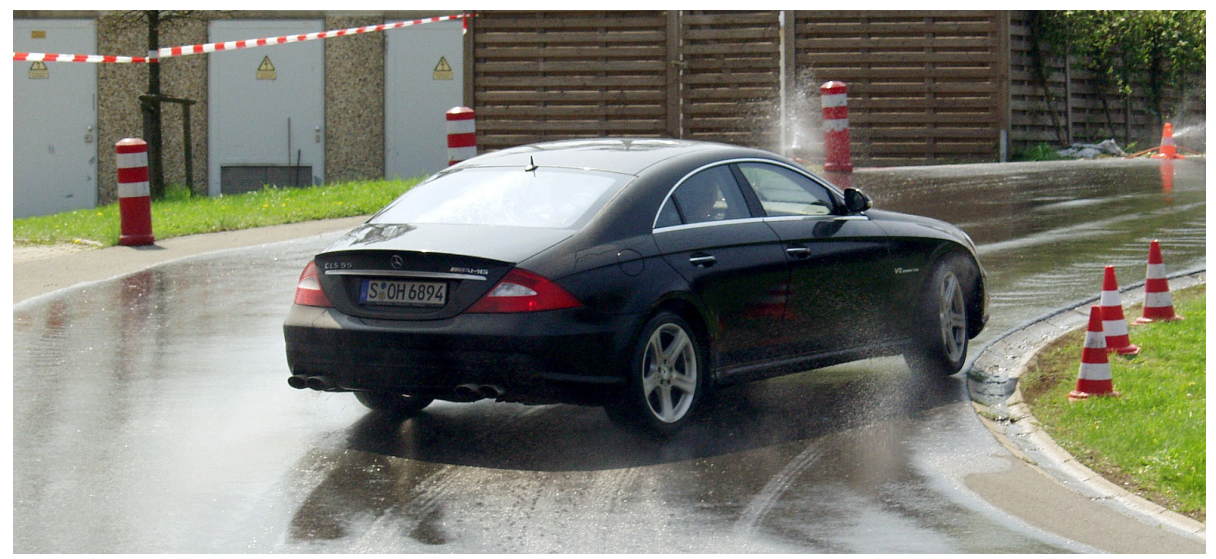

Figure 1.2: Car sliding on wet asphalt. Copyright (C) Raimond Spekking / CC BY-SA 4.0 (via Wikimedia Commons) [19].

\subsection{Motivation}

A necessary enabler of systems for assisted driving is the increased amount of sensors available in modern vehicles. The increased situational awareness required for assisted driving functions and future self-driving vehicles enables a new generation of autonomous active-safety systems. These use information about the location of obstacles, such as pedestrians and lane borders, not only to aid the driver in maintaining stability over the vehicle, but also to actively avoid obstacles. In self-driving vehicles, these obstacle aware active-safety functions help to increase the robustness of the self-driving system by handling critical situations, where comfort and other criteria secondary to safety are no longer of major importance.

To this end, the vehicle should utilize all available tire-road friction to minimize danger. Considering AEB, which given information about objects in front can initiate heavy braking without any driver command, there is the possibility to expand the capabilities of these systems to handle the more general task of collision avoidance by both braking and steering. Braking in a situation where only longitudinal dynamics has to be considered is relatively straightforward, where close to optimal performance can be achieved by relying on the ABS to keep the tire forces close to the limit of friction. When also lateral dynamics has a significant impact on the task of collision avoidance, perhaps requiring vehicle maneuvers similar to what is depicted in Figure 1.2, the problem becomes more complex. 
At a high level, the system needs to adaptively make a trade-off between controlling the longitudinal, lateral, and rotational dynamics of the vehicle in such a way that the risk of collision is minimized. The dynamics of a vehicle operating at the limit of friction is highly nonlinear and dependent on the in general unknown parameters characterizing the road. This is a challenge, both in terms of real-time planning and control under computational constraints, and in terms of real-time estimation of necessary parameters. In analogy with how the vehicle behavior under mild driving conditions can be explained by simplified models, it is of interest to find control principles that support the development of future active-safety systems capable of operating at the limit of friction. Optimal control has many applications related to vehicle dynamics [20-23], and can in this case be used to find control principles that capture the essential behavior of optimal vehicle maneuvering in safety-critical situations. Such control principles can lead to simplifications in the problems of planning, control, and parameter estimation for autonomous vehicle maneuvering at the limit of friction.

\subsection{Outline}

An introduction to the vehicle models used is given in Chapter 2 along with some examples of the models' usage in literature. The chapter concludes with a discussion on model usage from the perspective of the thesis. A summary of the papers in this thesis and their contributions are given in Chapter 3. The chapter ends with a reflection on the content of the thesis and areas of further exploration. The papers included in the thesis can be categorized into the three parts: analysis of optimal maneuvers, computing appropriate steering and braking commands from an acceleration-vector reference, and computing acceleration-vector references in challenging scenarios.

In Papers I-II, autonomous safety-critical maneuvers are analyzed using optimal control to gain insights into the optimal behavior. Paper I uses a linear combination of the initial and final velocity of the maneuver as the optimization criterion to investigate how the behavior depends on the level of braking. Paper II compares the optimal trajectories with the attainable forces to examine the trade-off between the forces acting on the vehicle.

In Papers III-IV, insights gained from the optimal behavior are used to develop controllers capable of operating at the limit of friction. These 
are based on following an acceleration-vector reference obtained using friction-limited particle models, and use this reference to compute appropriate steering and braking commands. Paper III introduces the controller structure used, while Papers IV expands on it to develop a controller for evasive maneuvering that does not use any estimation of the tire-road friction coefficient.

Finally, Papers V-VI handle computing of acceleration-vector references in challenging scenarios, which are used in acceleration-based controllers like those in Papers III-IV. Paper V considers a minimum-time lane change where a simple friction-limited particle model proves insufficient to provide a good reference, while Paper VI considers a general road with obstacles and uses a receding-horizon approach to generate the acceleration-vector reference.

\section{Licentiate Thesis}

In partial fulfillment of the Doctor's degree, a Licentiate Thesis was presented [24]. It contains earlier versions of Chapters 1-3 and Papers I-II, studying the optimal behavior in safety-critical vehicle maneuvers. Since then, the focus has been on real-time control with a clear connection to the research and ideas presented in the Licentiate Thesis. 


\section{Chapter 2}

\section{Vehicle Modeling}

$\mathrm{T}$ HIS chapter describes a number of models commonly used for vehicle motion control. Except for the higher-fidelity models available in the software CarMaker [25] used to verify controllers in simulation, this chapter includes descriptions of components composing all the models used in this thesis. The chapter aims to complement the papers included in this thesis with details about the models used and provide context for their use by highlighting their properties and giving examples of their usage in literature. The chapter starts off by describing different representations of the vehicle position, then continues with describing the different chassis models and tire models. Finally, the chapter concludes with a discussion about some of the model usage in the thesis.

\subsection{World Coordinate Systems}

Regardless of how the dynamics of the vehicle is modeled, it is important to relate its position to other objects in the world. There are two common ways to describe the vehicle position, in a global coordinate frame that is fixed with respect to the earth, or in a curvilinear road-aligned coordinate frame that turns along with a specified path. In this thesis, only flat roads without slopes are considered. 


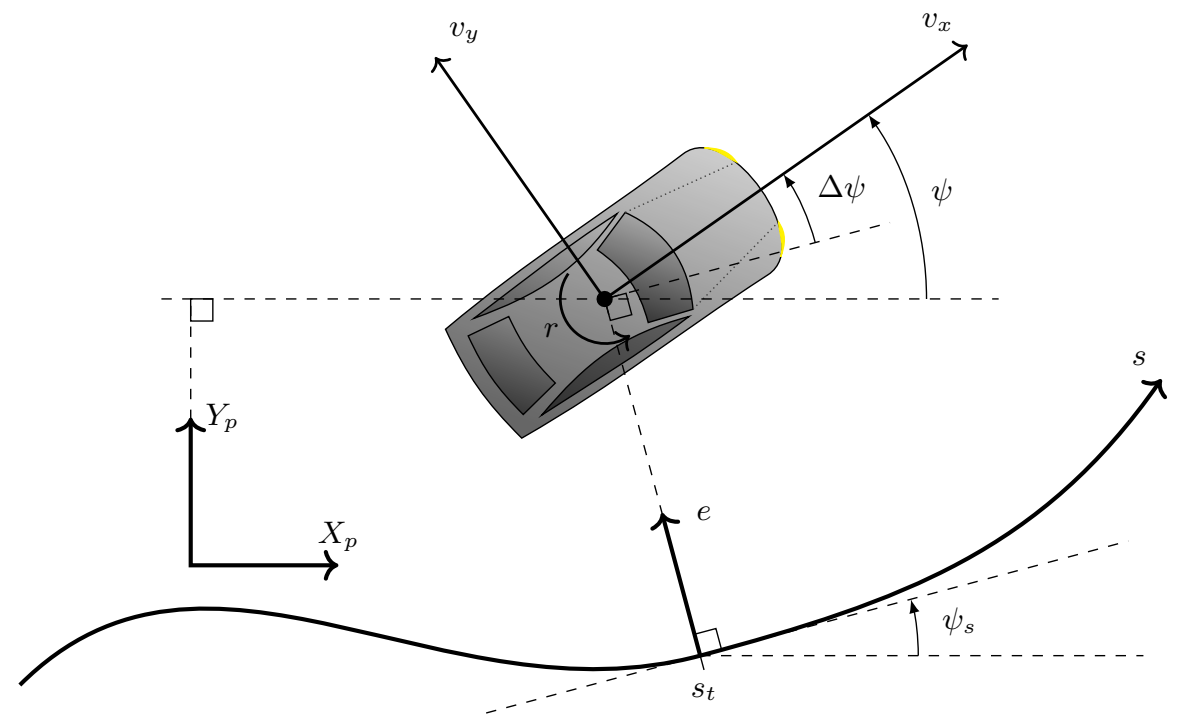

Figure 2.1: Vehicle position and orientation in the earth-fixed coordinates and in the road-aligned coordinates at the position $s=s_{t}$ along the specified path.

\subsubsection{Earth-Fixed Coordinate System}

In an earth-fixed coordinate system, the position $\left(X_{p}, Y_{p}\right)$ and orientation $\psi$ of the vehicle are, given an initial state, obtained from the differential equations

$$
\begin{aligned}
\dot{X}_{p} & =v_{x} \cos (\psi)-v_{y} \sin (\psi), \\
\dot{Y}_{p} & =v_{x} \sin (\psi)+v_{y} \cos (\psi), \\
\dot{\psi} & =r
\end{aligned}
$$

where $v_{x}$ is the longitudinal velocity, $v_{y}$ is the lateral velocity, and $r$ is the yaw rate as defined in Figure 2.1. By using the state variables $X_{p}$, $Y_{p}$, and $\psi$, the interaction of the vehicle with the world can be defined by putting constraints on these states of the form $f\left(X_{p}, Y_{p}, \psi\right) \leq 0$, e.g., to model the space outside lane borders or inside obstacles that the vehicle can not occupy.

For long vehicles such as buses, it becomes especially important to consider the constraints they impose because of their physical size [26]. This thesis considers passenger cars traveling at relatively high velocity, and the physical size is accounted for by enlarging the obstacle region, making it sufficient to impose constraints on the vehicle position $f\left(X_{p}, Y_{p}\right) \leq 0$. 
This representation is used in Papers I-II to model a constant-radius turn with limited lane width and a double lane-change track [27]. The earthfixed coordinate system gives simple dynamic equations, but can lead to a complicated representation of the obstacle region when compared to the road-aligned coordinate system. It is the natural representation when considering unstructured roads with possibly maze-like characteristics, see, e.g., $[28,29]$.

\subsubsection{Road-Aligned Coordinate System}

A path specification in the earth-fixed coordinate system can be defined by its starting position $\left(X_{s}(0), Y_{s}(0)\right)$, starting direction $\psi_{s}(0)$, and path curvature $\kappa(s)$ where $s$ specifies the distance along the path. The path can then be recovered from the differential relations

$$
\begin{aligned}
\frac{\mathrm{d} X_{s}}{\mathrm{~d} s} & =\cos \left(\psi_{s}\right), \\
\frac{\mathrm{d} Y_{s}}{\mathrm{~d} s} & =\sin \left(\psi_{s}\right), \\
\frac{\mathrm{d} \psi_{s}}{\mathrm{~d} s} & =\kappa(s) .
\end{aligned}
$$

In the road-aligned coordinate system, the vehicle position $(s, e)$ and orientation $\Delta \psi$ are used as defined in Figure 2.1. The equations of motion are not as straightforward to write down for this road-aligned coordinate system as it is for the earth-fixed coordinate system. To derive $\dot{s}$, expressions for the change of path direction $\dot{\psi}_{s}$ using the curvature and velocity obtained at the path centerline are compared with those obtained at the vehicle position. Denoting the parallel movement of the vehicle with respect to the tangent of the path (2.2) as $\dot{s}_{e}$ (see Figure 2.2), the corresponding curvature $\kappa_{e}(s, e)$ from the path-parallel movement is given by

$$
\kappa_{e}(s, e)=\frac{1}{R_{e}(s, e)}=\frac{1}{R(s)-e}=\frac{1}{\frac{1}{\kappa(s)}-e},
$$

where $R_{e}(s, e)$ and $R(s)$ are the radii of the curvatures $\kappa_{e}(s, e)$ and $\kappa(s)$, respectively (see Figure 2.2). The time derivative of $s$ can then be retrieved from:

$$
\begin{aligned}
& \dot{\psi}_{s}=\frac{\mathrm{d} \psi_{s}}{\mathrm{~d} t}=\frac{\mathrm{d} \psi_{s}}{\mathrm{~d} s} \frac{\mathrm{d} s}{\mathrm{~d} t}=\kappa(s) \dot{s}, \\
& \dot{\psi}_{s}=\frac{\mathrm{d} \psi_{s}}{\mathrm{~d} t}=\frac{\mathrm{d} \psi_{s}}{\mathrm{~d} s_{e}} \frac{\mathrm{d} s_{e}}{\mathrm{~d} t}=\kappa_{e}(s, e) \dot{s}_{e}=\frac{v_{x} \cos (\Delta \psi)-v_{y} \sin (\Delta \psi)}{\frac{1}{\kappa(s)}-e} .
\end{aligned}
$$




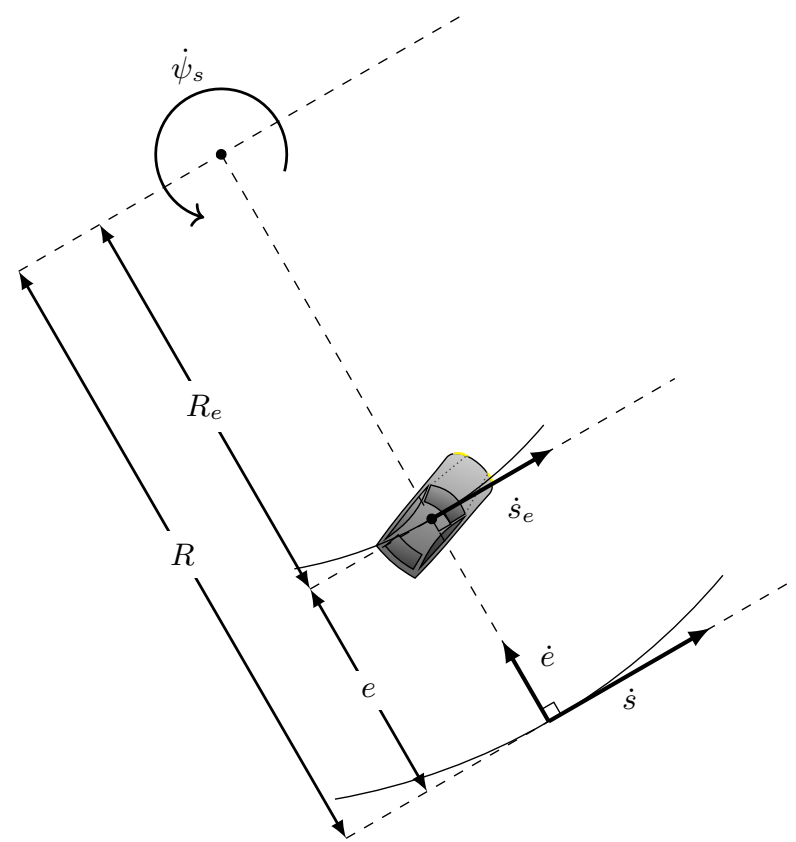

Figure 2.2: Kinematic relation between radii of curvatures $R$ and $R_{e}$ and the corresponding velocities $\dot{s}$ and $\dot{s}_{e}$ parallel to the tangent of the path.

The time derivative of the orientation $\Delta \psi$ can be calculated as

$$
\Delta \dot{\psi}=\dot{\psi}-\dot{\psi}_{s}=r-\kappa(s) \dot{s} .
$$

The resulting equations describing the motion in the road-aligned coordinate system are

$$
\begin{aligned}
\dot{s} & =\frac{v_{x} \cos (\Delta \psi)-v_{y} \sin (\Delta \psi)}{1-\kappa(s) e}, \\
\dot{e} & =v_{x} \sin (\Delta \psi)+v_{y} \cos (\Delta \psi), \\
\Delta \dot{\psi} & =r-\kappa(s) \dot{s} .
\end{aligned}
$$

While the equations $(2.7)$ are more complicated than those for the earth-fixed representation (2.1), the road-aligned coordinate system allows for a natural encoding of the lane boundaries as $e_{\min }(s) \leq e(s) \leq e_{\max }(s)$. In Paper VI, this representation is used to plan the motion of the vehicle. It is also used in [30] for online planning and control and in [31] to ensure affine lane boundaries. Similar representations are also used for path following, e.g., [32], where the error dynamics can be linearized around the path. 


\subsection{Chassis Models}

Depending on the type of vehicle and its configuration, different models of the chassis are required to capture its essential dynamics. Here, a typical car with four wheels is considered that can be modeled as a complex multibody system with individual dampers. Considering the planar motion studied in this thesis the complexity is limited to, in addition to the planar dynamics, also consider the influence of pitch and roll. The section starts with simpler chassis models where the forces between the tires and the road are not explicitly modeled. It then continues by describing chassis models that incorporate tire models such as those described in Section 2.3. Finally, it describes a model for aerodynamic resistance.

\subsubsection{Friction-Limited Particle Model}

The simplest representation of a vehicle is to disregard the fact that a vehicle cannot freely travel in any direction. This means that the longitudinal and lateral accelerations, $a_{x}$ and $a_{y}$, respectively, of the vehicle can be directly controlled as

$$
\begin{aligned}
& \dot{v}_{x}=a_{x}, \\
& \dot{v}_{y}=a_{y} .
\end{aligned}
$$

The orientation of the vehicle does in this case not limit its movement. While the above model can be used as is, in this thesis it is always used in conjunction with an acceleration constraint given by the limited friction $\mu$ between the vehicle and the road, and the gravitational acceleration constant $g$ :

$$
a_{x}^{2}+a_{y}^{2} \leq(\mu g)^{2},
$$

which is illustrated in Figure 2.3.

Even though this model is the simplest considered, it is a good representation of vehicle motion when operating at the limit of the tire-road friction. When operating in regions with high acceleration, the constraints on the acceleration become the limiting factor rather than limitations in, e.g., wheel angle. On the other hand, the model does not provide any relation for how the steering of the vehicle relates to its motion.

In this thesis, the friction-limited particle model is used to plan the vehicle motion in Papers III-VI. Other constraints than (2.10) on the accelerations can be used to model for example effects of load transfer and limited engine power [33]. An advantage of the friction-limited particle 


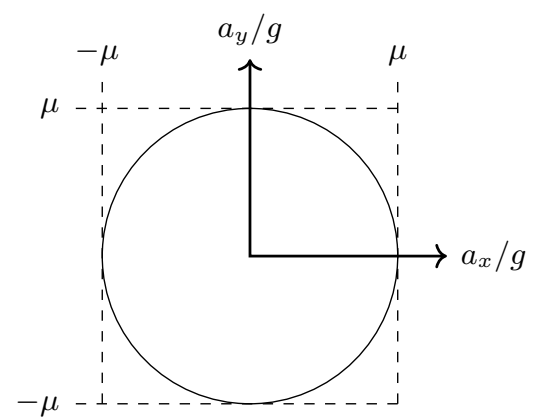

Figure 2.3: Illustration of the friction limit (2.10).

model is that for some scenarios there exist analytical solutions [34-36] and in other scenarios the solutions can be efficiently computed [37, 38].

\subsubsection{Kinematic Model}

The kinematic vehicle model is not used in this thesis, but is presented for completeness considering its common use in literature. By assuming that the velocity of each wheel is parallel to the wheel's ego direction, a purely kinematic model describing the lateral vehicle motion is obtained, meaning that it does not contain derivatives of the velocities $v_{x}, v_{y}$, and $r$. This model is commonly referred to as the kinematic bicycle model or to as the kinematic single-track model, with the model typically being described by only one wheel on each axle. In the Ackermann steering geometry [39], the inner front wheel has a larger steering angle than the outer wheel, such that none of the wheels has lateral movement. As illustrated in Figure 2.4, the resulting equations for the kinematic singletrack model will also describe the equivalent vehicle with four wheels with Ackermann steering geometry.

With the kinematic single-track model, the rear wheel is commonly used as the reference point. For the rear wheels to have no lateral movement, this leads to

$$
\begin{aligned}
& v_{x}=v, \\
& v_{y}=0 .
\end{aligned}
$$




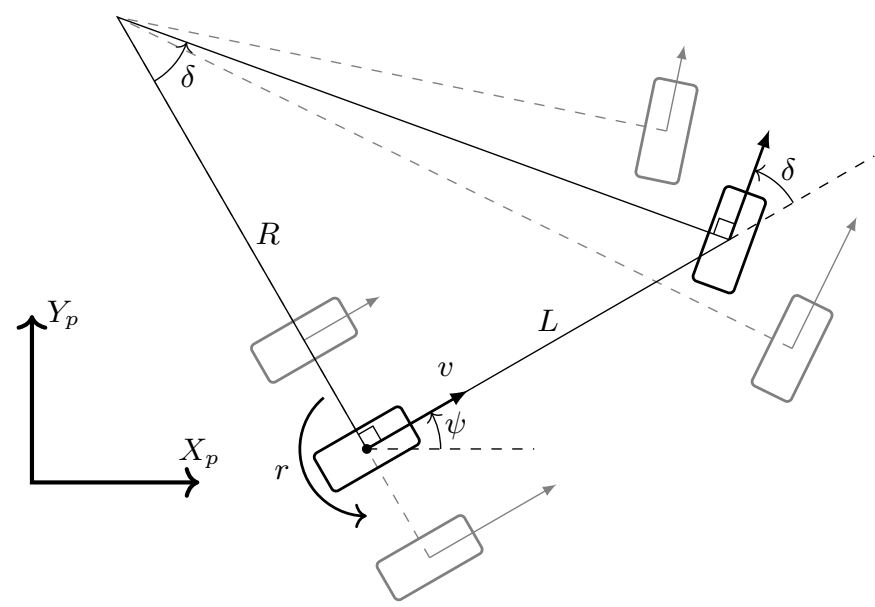

Figure 2.4: Kinematic model with illustration of Ackermann steering.

A relation between the steering angle $\delta$ and the yaw rate $r$ can be found from the geometry in Figure 2.4 as

$$
r=\frac{v}{R}=v \frac{\tan (\delta)}{L} .
$$

The model can now be used in either coordinate system (2.1) or (2.7). Using the earth-fixed coordinate system (2.1) gives

$$
\begin{aligned}
\dot{X}_{p} & =v \cos (\psi), \\
\dot{Y}_{p} & =v \sin (\psi), \\
\dot{\psi} & =v \frac{\tan (\delta)}{L},
\end{aligned}
$$

If the distance traveled by the vehicle is denoted by $s$ and replace the time $t$ as the free variable in (2.12), then the path traveled by the vehicle is obtained from

$$
\begin{gathered}
\frac{\mathrm{d} X_{p}}{\mathrm{~d} s}=\frac{\mathrm{d} X_{p}}{\mathrm{~d} t} \frac{\mathrm{d} t}{\mathrm{~d} s}=\frac{\dot{X}_{p}}{v}=\cos (\psi), \\
\frac{\mathrm{d} Y_{p}}{\mathrm{~d} s}=\frac{\mathrm{d} Y_{p}}{\mathrm{~d} t} \frac{\mathrm{d} t}{\mathrm{~d} s}=\frac{\dot{Y}_{p}}{v}=\sin (\psi), \\
\frac{\mathrm{d} \psi}{\mathrm{d} s}=\frac{\mathrm{d} \psi}{\mathrm{d} t} \frac{\mathrm{d} t}{\mathrm{~d} s}=\frac{\dot{\psi}}{v}=\frac{\tan (\delta)}{L},
\end{gathered}
$$


which is independent of the velocity $v$. Further, if the curvature of the vehicle path is denoted by $\kappa$ and is used as a virtual input

$$
\tan (\delta)=\frac{L}{R}=L \kappa,
$$

then the resulting control-affine system is equivalent to the path definition (2.2). Because of this, it is typical in the application of this model to decouple the tasks of longitudinal and lateral motion planning in a pathvelocity decomposition [40]. For example, a path can first be planned using (2.13), afterwards the velocity can be planned with respect to, e.g., acceleration limits [41].

Using the kinematic model to generate a set of motion primitives, it can be used for efficient lattice-based path planning with $A^{*}[42]$. With its no-slip assumption limiting the accuracy at higher lateral accelerations, the kinematic model is suitable for low-velocity scenarios such as parking [43], but it has also successfully been used for control in scenarios with moderate accelerations [44].

\subsubsection{Single-Track Model}

It is now considered that the vehicle can have an orientation and rotation independent of the vehicle path. To simplify, it is common to lump the tires on the same axle together, which is often referred to as the singletrack model or the bicycle model. At steady-state, this can be compared to the kinematic model, but now the turning radius is influenced by the slip angles $\alpha_{f}$ and $\alpha_{r}$ of the front and rear tires. The kinematics described in Figure 2.5 gives the approximate relation [39]:

$$
\delta \approx \frac{L}{R}+\alpha_{f}-\alpha_{r}
$$

which can be compared with the relation for the kinematic model (2.14).

These slip angles give rise to the lateral tire forces $F_{y, f}$ and $F_{y, r}$ at the front and rear wheel, respectively (see Figure 2.6). Together with the longitudinal tire forces $F_{x, f}$ and $F_{x, r}$, these forces determine the motion of the vehicle. Different models of the relation between the tire slips and the tire forces are described in Section 2.3. The total forces and moment acting on the vehicle center of mass are given by:

$$
\begin{aligned}
F_{x} & =\cos (\delta) F_{x, f}-\sin (\delta) F_{y, f}+F_{x, r}, \\
F_{y} & =\cos (\delta) F_{y, f}+\sin (\delta) F_{x, f}+F_{y, r}, \\
M_{z} & =l_{f} \cos (\delta) F_{y, f}+l_{f} \sin (\delta) F_{x, f}-l_{r} F_{y, r} .
\end{aligned}
$$




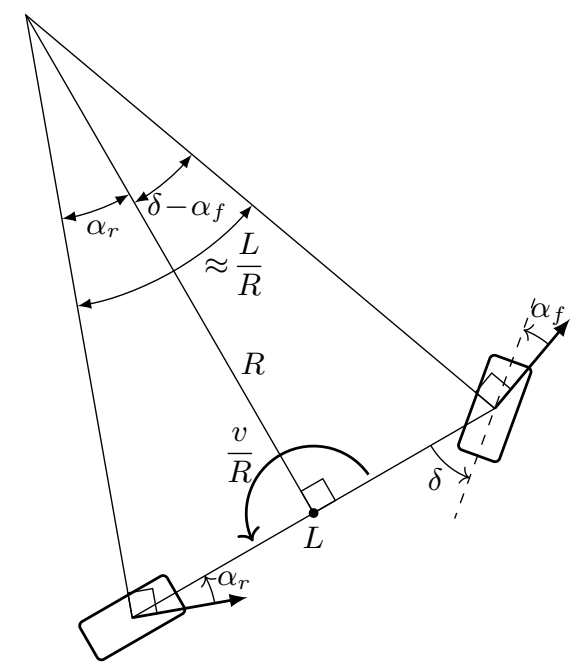

Figure 2.5: Single-track model during steady-state cornering.

In an inertial frame of reference, Newton's second law applied on the forces and moments (2.16) gives rise to the accelerations

$$
\begin{aligned}
a_{x} & =\frac{F_{x}}{m}, \\
a_{y} & =\frac{F_{y}}{m}, \\
\varepsilon & =\frac{M_{z}}{I_{z z}},
\end{aligned}
$$

where $a_{x}$ is the longitudinal acceleration, $a_{y}$ is the lateral acceleration, $\varepsilon$ is the yaw acceleration, $m$ is the vehicle mass, and $I_{z z}$ is the yaw moment of inertia.

Since the noninertial coordinate frame of the vehicle is moving and rotating, the relative velocities will differ when compared with the inertial global coordinate frame, and so will also the relative accelerations. Denoting the unit vectors of the vehicle coordinate frame as $e_{x}$ and $e_{y}$, and the unit vectors of the global coordinate frame as $e_{X_{p}}$ and $e_{Y_{p}}$, the transformation between these are dependent on the vehicle orientation $\psi$ (see Figure 2.1) as

$$
\begin{aligned}
& e_{x}=e_{X_{p}} \cos (\psi)+e_{Y_{p}} \sin (\psi), \\
& e_{y}=-e_{X_{p}} \sin (\psi)+e_{Y_{p}} \cos (\psi) .
\end{aligned}
$$



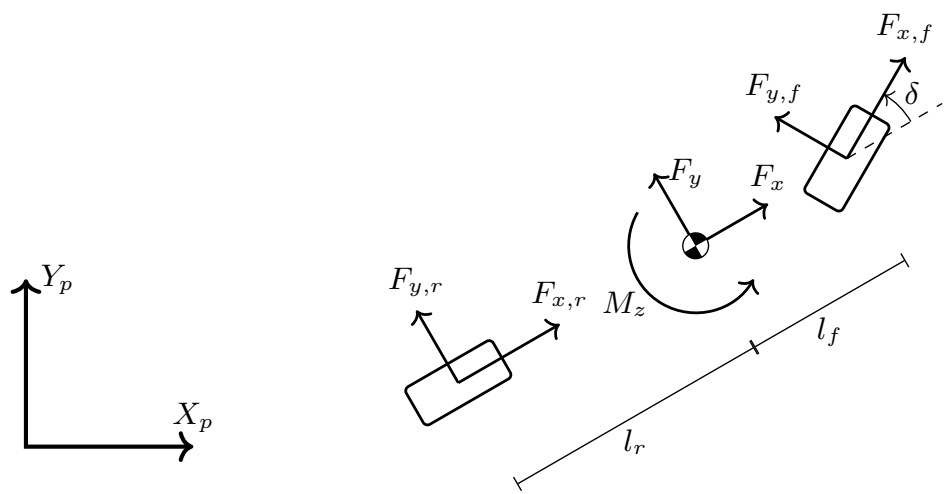

Figure 2.6: Single-track model.

The time derivatives of $e_{x}$ and $e_{y}$ in the inertial global coordinate frame are

$$
\begin{aligned}
& \dot{e}_{x}=-e_{X_{p}} \dot{\psi} \sin (\psi)+e_{Y_{p}} \dot{\psi} \cos (\psi)=r e_{y}, \\
& \dot{e}_{y}=-e_{X_{p}} \dot{\psi} \cos (\psi)-e_{Y_{p}} \dot{\psi} \sin (\psi)=-r e_{x} .
\end{aligned}
$$

Given the velocity $v_{x} e_{x}+v_{y} e_{y}$, the acceleration is computed by

$$
\begin{aligned}
a_{x} e_{x}+a_{y} e_{y} & =\frac{\mathrm{d}}{\mathrm{d} t}\left(v_{x} e_{x}+v_{y} e_{y}\right) \\
& =\dot{v}_{x} e_{x}+v_{x} \dot{e}_{x}+\dot{v}_{y} e_{y}+v_{y} \dot{e}_{y} \\
& =\dot{v}_{x} e_{x}+v_{x} r e_{y}+\dot{v}_{y} e_{y}-v_{y} r e_{x} .
\end{aligned}
$$

Collecting the terms in front of each unit vector $e_{x}$ and $e_{y}$, the resulting equations of motion for the center of mass are ${ }^{1}$

$$
\begin{aligned}
\dot{v}_{x}=a_{x}+v_{y} r & =\frac{F_{x}}{m}+v_{y} r, \\
\dot{v}_{y}=a_{y}-v_{x} r & =\frac{F_{y}}{m}-v_{x} r, \\
\dot{r} & =\varepsilon \quad=\frac{M_{z}}{I_{z z}} .
\end{aligned}
$$

\footnotetext{
${ }^{1}$ In general, given a reference frame and another frame rotating relative to this reference frame with the angular velocity $\boldsymbol{\omega}$, the rate of change of a vector $\mathbf{u}$ observed in each frame is related by [45]:

$$
\left.\frac{\mathrm{d} \mathbf{u}}{\mathrm{d} t}\right|_{\text {reference }}=\left.\frac{\mathrm{d} \mathbf{u}}{\mathrm{d} t}\right|_{\text {rotating }}+\boldsymbol{\omega} \times \mathbf{u}
$$
}


Because the tire traction is heavily dependent on the vertical tire loads [39], it is important to consider these. Let $h$ denote the vertical position of the center of mass above the ground plane, then the vertical tire forces $F_{z, f}$ and $F_{z, r}$ of the front wheel and the rear wheel, respectively, are governed by the vertical force and pitch-moment equilibria

$$
\begin{aligned}
F_{z, f}+F_{z, r} & =m g, \\
l_{f} F_{z, f}-l_{r} F_{z, r} & =h F_{x},
\end{aligned}
$$

where the vehicle is assumed to be a rigid body. By calculating the moment equilibrium around each wheel, the vertical tire forces are calculated explicitly to

$$
\begin{aligned}
& F_{z, f}=\frac{m g l_{r}+h F_{x}}{L}, \\
& F_{z, r}=\frac{m g l_{f}-h F_{x}}{L},
\end{aligned}
$$

where the first term is the static load distribution, and the second term is resulting from braking or accelerating and can be estimated by measuring the longitudinal acceleration $a_{x}\left(F_{x}=m a_{x}\right)$.

Compared to the kinematic vehicle model, the single-track model requires modeling of the tire forces as a function of the slip angles $\alpha_{f}$ and $\alpha_{r}$, which comes at the gain of much increased model accuracy at higher lateral accelerations. The single-track vehicle model is often able to accurately capture the behavior of the vehicle [46]. While the steadystate cornering behavior can be incorporated in the parameterization of the dynamic single-track model, other phenomena such as differential braking cannot. In this thesis, the rear wheels are lumped together in a single-track like simplification when determining references for the yaw controllers developed in Papers III, IV, and VI. It is a suitable model choice when the lateral dynamics of $v_{y}$ and $r$ are assumed to not be heavily influenced by the longitudinal dynamics. Examples of its use can be found in numerous designs of lateral control systems, e.g., [47-51].

\subsubsection{Double-Track Model}

In the double-track model, the forces from each of the four wheels are explicitly modeled. Each tire influences the chassis dynamics by its interaction with the road, see Section 2.3. Counted from left to right and front to rear in the vehicle ego orientation, the vehicle has four wheels 

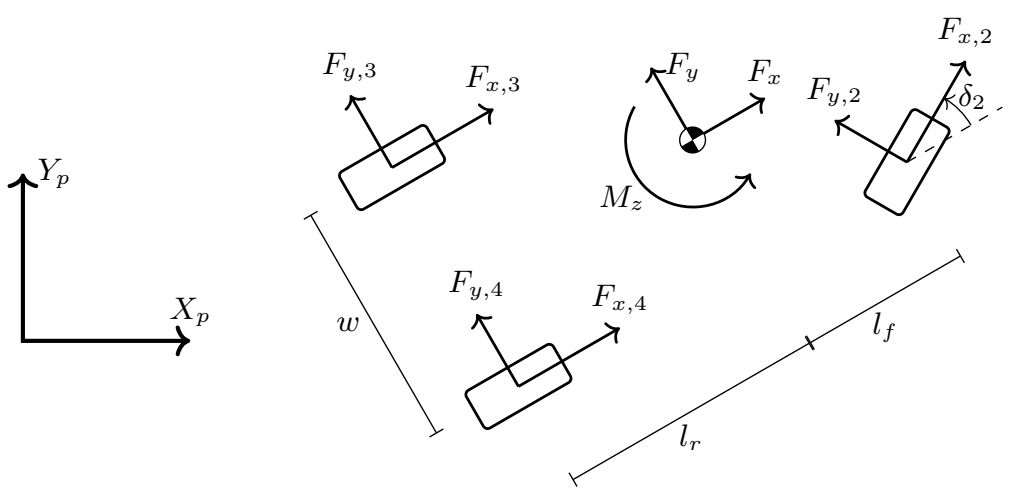

Figure 2.7: Double-track model.

denoted with subscript $i \in\{1,2,3,4\}$, see Figure 2.7. The variables $F_{x, i}$ and $F_{y, i}$ denote the force components from the tire traction of each wheel $i$ in the local coordinate system of the wheel, which is determined by the steering angle $\delta_{i}$ of the wheel relative to the vehicle body. Each wheel $i$ is fixed in the ground plane, at a longitudinal and lateral position $\left(l_{x, i}, l_{y, i}\right)$ from the center of mass. For example, for the double-track model illustrated in Figure 2.7, the wheel positions are given by $l_{x, 1}=l_{x, 2}=l_{f}$, $l_{x, 3}=l_{x, 4}=-l_{r}, l_{y, 1}=l_{y, 3}=w / 2$, and $l_{y, 2}=l_{y, 4}=-w / 2$. Written in matrix form for compactness, the total forces and moments acting on the vehicle center of mass are given by:

$$
\left[\begin{array}{c}
F_{x} \\
F_{y} \\
M_{z}
\end{array}\right]=\sum_{i=1}^{4}\left[\begin{array}{cc}
1 & 0 \\
0 & 1 \\
-l_{y, i} & l_{x, i}
\end{array}\right]\left[\begin{array}{cc}
\cos \left(\delta_{i}\right) & -\sin \left(\delta_{i}\right) \\
\sin \left(\delta_{i}\right) & \cos \left(\delta_{i}\right)
\end{array}\right]\left[\begin{array}{c}
F_{x, i} \\
F_{y, i}
\end{array}\right] .
$$

Like for the single-track model, the resulting equations of motion for the center of mass are then given by (2.21).

The steering angles of the rear wheels are zero (though there are cars with rear-wheel steering), i.e.,

$$
\delta_{3}=\delta_{4}=0
$$


On real cars, the steering-wheel angle influences the angles of the front wheels differently, typically in accordance with Ackermann steering geometry [39] (described for the kinematic model in Figure 2.4). A simplifying assumption is that the front wheels have the same wheel angle, $\delta$, according to

$$
\delta_{1}=\delta_{2}=\delta
$$

This simplification has little effect on the driving performance [52]. The vertical tire loads $F_{z, i}$ influence the tire traction and are modeled to fulfill the vertical force and moment equilibria

$$
\begin{gathered}
\sum_{i=1}^{4} F_{z, i}=m g, \\
\sum_{i=1}^{4} l_{x, i} F_{z, i}=-h F_{x}, \\
\sum_{i=1}^{2} l_{y, i} F_{z, i}=-\frac{h F_{y}}{2}, \\
\sum_{i=3}^{4} l_{y, i} F_{z, i}=-\frac{h F_{y}}{2},
\end{gathered}
$$

where there is some freedom in modeling how the lateral load transfer interacts with the longitudinal load transfer by selection of the last two equations. This system can be solved explicitly for each vertical load $F_{z, i}$. Assuming that the wheels are located as in Figure 2.7, the vertical load at each wheel is given by

$$
\begin{aligned}
& F_{z, 1}=\frac{m g w l_{r}-h w F_{x}-h L F_{y}}{2 w L}, \\
& F_{z, 2}=\frac{m g w l_{r}-h w F_{x}+h L F_{y}}{2 w L}, \\
& F_{z, 3}=\frac{m g w l_{f}+h w F_{x}-h L F_{y}}{2 w L}, \\
& F_{z, 4}=\frac{m g w l_{f}+h w F_{x}+h L F_{y}}{2 w L} .
\end{aligned}
$$

The double-track model is extensively used in this thesis because of its ability to model differential braking, which is heavily influenced by the lateral load transfer [53]. Some uses for the double-track model are studying the effects of differential braking [54] or torque vectoring [31], and when utilizing differential braking for control [55]. 


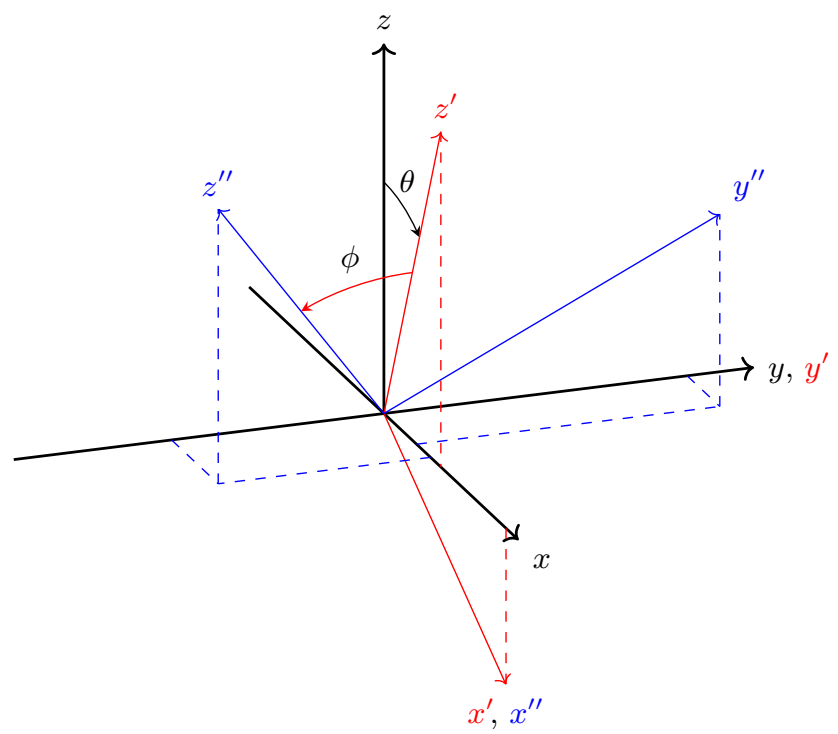

Figure 2.8: Coordinate transform from vehicle coordinates to vehicle-body coordinates. In this figure, $x$ is the longitudinal axis of the vehicle frame, $y$ is the lateral axis of the vehicle frame, and $z$ is the vertical axis of the vehicle frame. The pitch angle $\theta$ is the rotation around the $y$-axis and the roll angle $\phi$ is the rotation around the transformed $x^{\prime}$-axis.

\subsubsection{Five Degrees-of-Freedom Model}

Here the addition of pitch and roll dynamics are considered as derived in [56], resulting in two additional degrees of freedom. The pitch and roll are governed by rotational springs and dampers that model the effect of the suspension system. While the pitch and roll centers of rotation on real cars are located at different positions, this is simplified such that the vehicle position $\left(X_{p}, Y_{p}\right)$ marks the center of rotation in the vehicle frame and is fixed in the ground plane. The vehicle center of mass is located at a fixed distance $h$ from this point, with its position dependent on both the pitch and the roll. The pitch angle $\theta$ and the roll angle $\phi$ are defined in Figure 2.8 as the coordinate transformation from the vehicle frame $(x, y, z)$, to the suspended vehicle body frame $\left(x^{\prime \prime}, y^{\prime \prime}, z^{\prime \prime}\right)$. The position of the vehicle center of mass is constant in the fixed frame of the vehicle body, positioned a distance $h$ along the $z^{\prime \prime}$-axis in Figure 2.8. The suspension is modeled as independent spring-damper systems acting on the rotational degrees of freedom $\theta$ and $\phi$. This modeling means that coupled effects 
of the suspension system from combined roll and pitch motion are not taken into account, but for the purpose of this thesis it is sufficient to model the characteristics of the investigated load-transfer effects.

A summary of the motion equations, derived by Berntorp [56], is given below. The reader is referred to that reference for further details regarding the derivation of the model. From the moving coordinate systems of the vehicle in Figure 2.7, and the roll and pitch motion illustrated in Figure 2.8, the planar equations of motion can be written as

$$
\begin{aligned}
\dot{v}_{x}= & \frac{F_{x}}{m}+v_{y} r-h f_{x}, \\
\dot{v}_{y}= & \frac{F_{y}}{m}-v_{x} r-h f_{y}, \\
\dot{r}= & \frac{M_{z}+h \tau_{r}}{I_{r}}, \\
f_{x}= & \sin (\theta) \cos (\phi)\left(r^{2}+\dot{\phi}^{2}+\dot{\theta}^{2}\right)-\sin (\theta) \dot{r}-2 \cos (\phi) \dot{\phi} r \\
& +\sin (\theta) \sin (\phi) \dot{\phi} r-\sin (\phi) \dot{\phi}^{2}+\cos (\phi) \ddot{\phi}, \\
f_{y}= & -\sin (\theta) \cos (\phi) \dot{r}-\sin (\phi) r^{2}-2 \cos (\theta) \cos (\phi) \dot{\theta} r+ \\
& +\sin (\theta) \sin (\phi) \dot{\phi} r-\sin (\phi) \dot{\phi}^{2}+\cos (\phi) \ddot{\phi}, \\
\tau_{r}= & F_{x} \sin (\phi)+F_{y} \sin (\theta) \cos (\phi), \\
I_{r}= & I_{x x} \sin ^{2}(\theta)+\cos ^{2}(\theta)\left(I_{y y} \sin ^{2}(\phi)+I_{z z} \cos ^{2}(\phi)\right),
\end{aligned}
$$

where $h f_{x}$ and $h f_{y}$ are the contributions to $\dot{v}_{x}$ and $\dot{v}_{y}$ from the roll and pitch dynamics, $I_{r}$ is the moment of inertia associated with $r, h \tau_{r}$ is the additional external yaw moment from the deflection of the center of mass, $I_{x x}$ is the roll moment of inertia in the fixed frame of the vehicle body, $I_{y y}$ is the pitch moment of inertia in the fixed frame of the vehicle body, and $I_{z z}$ is the yaw moment of inertia in the fixed frame of the vehicle body. The equation of motion for the pitch dynamics is given by

$$
\begin{aligned}
\ddot{\theta} I_{\theta}+\nu_{\theta}= & -\left(D_{\theta} \dot{\theta}+K_{\theta} \theta\right)+h \tau_{\theta}, \\
I_{\theta}= & I_{y y} \cos ^{2}(\phi)+I_{z z} \sin ^{2}(\phi), \\
\nu_{\theta}= & r\left(r \sin (\theta) \cos (\theta)\left(\Delta I_{x y}+\cos ^{2}(\phi) \Delta I_{y z}\right)\right. \\
& -\dot{\phi} \cos ^{2}(\theta) I_{x x}+\sin ^{2}(\phi) \sin ^{2}(\theta) I_{y y} \\
& \left.+\sin ^{2}(\theta) \cos ^{2}(\phi) I_{z z}-\dot{\theta}\left(\sin (\theta) \sin (\phi) \cos (\phi) \Delta I_{y z}\right)\right), \\
\tau_{\theta}= & m g \sin (\theta) \cos (\phi)-F_{x} \cos (\theta) \cos (\phi),
\end{aligned}
$$


where $I_{\theta}$ is the moment of inertia associated with $\theta, \nu_{\theta}$ is the lowerorder inertial terms, $D_{\theta} \dot{\theta}+K_{\theta} \theta$ models a spring-damper system in the $\theta$-direction, and $h \tau_{\theta}$ is the external torques from the deflection of the center of mass. The equation of motion for the roll dynamics is given by

$$
\begin{aligned}
\ddot{\phi} I_{\phi}+\nu_{\phi}= & -\left(D_{\phi} \dot{\phi}+K_{\phi} \phi\right)+h \tau_{\phi}, \\
I_{\phi}= & I_{x x} \cos ^{2}(\theta)+I_{y y} \sin ^{2}(\theta) \sin ^{2}(\phi)+I_{z z} \sin ^{2}(\theta) \cos ^{2}(\phi), \\
\nu_{\phi}= & r \Delta I_{y z}(r \sin (\phi) \cos (\phi) \cos (\theta)+\dot{\phi} \sin (\theta) \sin (\phi) \cos \phi) \\
& +r \dot{\theta}\left(I_{y y} \cos ^{2}(\phi)+I_{z z} \sin ^{2}(\phi)\right), \\
\tau_{\phi}= & m g \sin (\phi)+F_{y} \cos (\phi) \cos (\theta),
\end{aligned}
$$

where $I_{\phi}$ is the moment of inertia associated with $\phi, \nu_{\phi}$ is the lowerorder inertial terms, $D_{\phi} \dot{\phi}+K_{\phi} \phi$ models a spring-damper system in the $\phi$-direction, and $h \tau_{\phi}$ is the external torques from the deflection of the center of mass.

The vertical tire load on each wheel, $F_{z, i}$, influences the tire traction and can be solved from force and moment equilibria. Applied to the double-track model in Section 2.7, these are given by

$$
\begin{aligned}
\sum_{i=1}^{4} F_{z, i} & =m g \\
\sum_{i=1}^{4} l_{x, i} F_{z, i} & =K_{\theta} \theta+D_{\theta} \dot{\theta}, \\
\sum_{i=1}^{2} l_{y, i} F_{z, i} & =K_{\phi} \phi / 2+D_{\phi} \dot{\phi} / 2, \\
\sum_{i=3}^{4} l_{y, i} F_{z, i} & =K_{\phi} \phi / 2+D_{\phi} \dot{\phi} / 2 .
\end{aligned}
$$

and will result in similar tire loads as the static-load transfer relations (2.27) when at steady state. Compared to those relations, (2.32) captures transients and dynamic effects in the vertical forces because of the suspension system, and the effects from the deflection of the center of mass.

\subsubsection{Aerodynamic Resistance}

When driving at speed, the aerodynamic resistance results in a force that slows the car down, and cross winds can influence the lateral motion 
of the car. Here, it is primarily the former that is addressed to enable direct comparisons with high-fidelity models such as those available in CarMaker [25]. The aerodynamic resistance $R_{a}$ can be expressed by [39]

$$
R_{a}=\frac{\rho}{2} C_{D} A_{f} v_{r}^{2}
$$

where $\rho$ is the mass density of the air, $C_{D}$ is the coefficient of aerodynamic resistance, $A_{f}$ is the characteristic area of the vehicle, and $v_{r}$ is the speed of the vehicle relative to the wind. This model of the aerodynamic resistance is used in Papers IV-VI under some simplifying assumptions. Assuming that the wind speed is zero, (2.33) gives the longitudinal and lateral aerodynamic resistances

$$
\begin{aligned}
& R_{a, x}=K_{D, x} v_{x} \sqrt{v_{x}^{2}+v_{y}^{2}}, \\
& R_{a, y}=K_{D, y} v_{y} \sqrt{v_{x}^{2}+v_{y}^{2}},
\end{aligned}
$$

where the aerodynamic parameters are lumped together into the parameters $K_{D, x}$ and $K_{D, y}$. Assuming that the aerodynamic resistance force acts on the center of mass so that the moments are not influenced, these can be incorporated into the vehicle model by replacing $F_{x}$ and $F_{y}$ in (2.24) with $F_{x}-R_{a, x}$ and $F_{y}-R_{a, y}$, respectively.

In this thesis, the longitudinal velocity $v_{x}$ is typically much larger than $v_{y}$, leading to the lateral velocity having little effect on the total aerodynamic resistance. To simplify, the aerodynamic parameters are approximated to be the same in the longitudinal and lateral directions $K_{D, x}=K_{D, y}=K_{D}$. As mentioned initially, the main purpose of including aerodynamics in this thesis is to achieve similar velocity decreases as in CarMaker to enable comparison, and for that purpose the even simpler model $R_{a, x}=K_{D} v_{x}^{2}$ is sufficient.

\subsection{Wheel and Tire Modeling}

The tire model employed is an important component determining the overall vehicle behavior. They come in a wide range of complexity, where more complex models aim at capturing high-frequency phenomena and are used to analyze comfort and noise [57]. Here, to capture the essential dynamics governing maneuvering, steady-state tire models are employed. An effect not directly modeled is the force resulting from the camber angle, the angle with which the wheel leans. Instead its steady-state effects on 
the vehicle are included in the parameterization of the lateral tire-force models.

First, the rotational wheel dynamics are described, then common definitions for tire slips that are used when modeling tire forces are described. After that some tire models for pure slip conditions are described, which are valid for a tire experiencing only purely longitudinal or purely lateral tire traction. Finally, models of the behavior under combined slip conditions are described.

\subsubsection{Wheel Dynamics}

The rotational dynamics of a wheel is illustrated in Figure 2.9(a). By adding up the total torque contribution to the wheel rotation, the dynamics for the wheel states, i.e., the rotational speeds $\omega_{i}$, are given by

$$
I_{w} \dot{\omega}_{i}=T_{i}-R_{e} F_{x, i}
$$

where $T_{i}$ is the applied driving or braking torque on wheel $i, I_{w}$ is the rotational wheel inertia, and $R_{e}$ is the effective wheel radius.

To model a brake system in Papers I-II, a first-order system with time constant $\gamma_{T}$ is used, where the braking of each wheel is individually actuated by the commanded torque $T_{u, i}$ :

$$
\gamma_{T} \dot{T}_{i}+T_{i}=T_{u, i}
$$

In this thesis only braking is considered. If both braking and driving torques are to be considered, different time constants are appropriate to reflect the different dynamics.

\subsubsection{Tire Slip}

The tire-road interaction is dependent on the movement of the tire's contact patch with the road. The velocity of each wheel in its local coordinate system, $\left(v_{x, i}, v_{y, i}\right)$, is described by

$$
\left[\begin{array}{l}
v_{x, i} \\
v_{y, i}
\end{array}\right]=\left[\begin{array}{cc}
\cos \left(\delta_{i}\right) & \sin \left(\delta_{i}\right) \\
-\sin \left(\delta_{i}\right) & \cos \left(\delta_{i}\right)
\end{array}\right]\left(\left[\begin{array}{c}
v_{x} \\
v_{y}
\end{array}\right]+r\left[\begin{array}{c}
-l_{y, i} \\
l_{x, i}
\end{array}\right]\right) .
$$

To model the tire-road interaction, the definitions from [58] are used to 


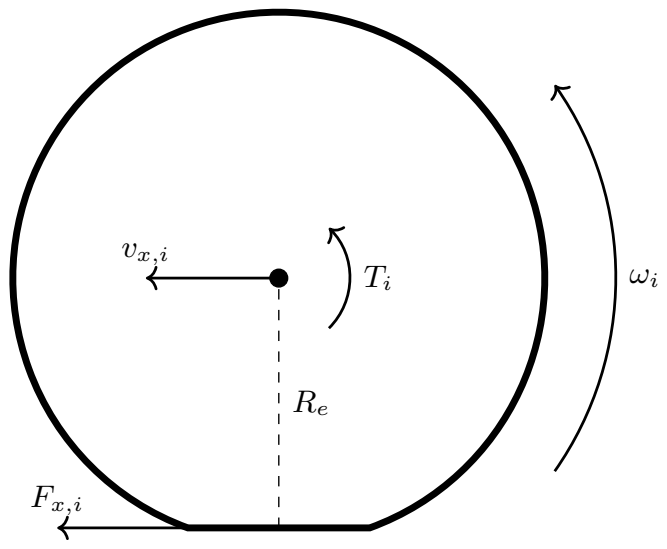

(a) Side view.

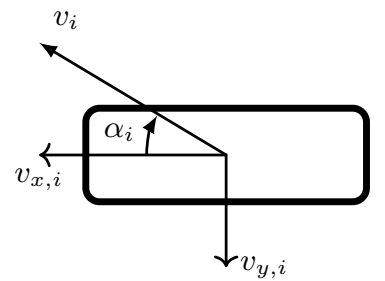

(b) Top view.

Figure 2.9: Wheel model.

describe the longitudinal tire slip and the lateral tire slip. The longitudinal slip ratios $\kappa_{i}$ are defined as

$$
\kappa_{i}=\frac{R_{e} \omega_{i}-v_{x, i}}{v_{x, i}} .
$$

With this definition, a locked wheel has the slip ratio $\kappa_{i}=-1$ and a free-rolling wheel has the slip ratio $\kappa_{i}=0$. The lateral slip angles $\alpha_{i}$ are defined as the angle between the wheel ego direction and the velocity vector $v_{i}$ of the wheel, see Figure 2.9(b), i.e.,

$$
\alpha_{i}=-\arctan \left(\frac{v_{y, i}}{v_{x, i}}\right) .
$$

To account for the transient behavior in the tire where it takes some time for it to deform, the relaxation length $\sigma$ is used, which is the longitudinal distance traveled by the tire before the lateral tire forces are at steadystate. This transient extension of the otherwise steady-state tire models used is defined by [58]

$$
\frac{\sigma}{v_{x, i}} \dot{\alpha}_{i}+\alpha_{i}=-\arctan \left(\frac{v_{y, i}}{v_{x, i}}\right) .
$$


There are alternative slip definitions such as [59]

$$
\begin{aligned}
\sigma_{x} & =\frac{v_{x, i}-R_{e} \omega_{i}}{R_{e} \omega_{i}}, \\
\sigma_{y} & =\frac{v_{y, i}}{R_{e} \omega_{i}},
\end{aligned}
$$

which are physically motivated by their correspondence to the deformation of the tire. These are used in Paper IV when considering the modeling of combined slip. For braking (which is a focus in this thesis), they are not as convenient as (2.38) and (2.39) because of the singularity at wheel lock $\left(\omega_{i}=0\right)$.

\subsubsection{Brush Tire Model}

In the brush tire models, the tire thread elements are modeled as elastic bristles on a brush, which leads to both adhesive forces from the brush elements' extension, and to sliding forces when the brush elements lose their grip and start sliding [58]. This gives rise to an adhesive region and a sliding region on the contact patch between the tire and the road. With the contact patch extending $\pm a_{i}$ in the wheel ego direction, the adhesive region stretches from the front of the contact patch $a_{i}$ to a point $x_{s t, i}$ where the thread elements start sliding, and the sliding region stretches from $x_{s t, i}$ to a point $-a_{i}$. The resulting lateral tire force is computed from

$$
F_{y 0, i}=\int_{-a_{i}}^{x_{s t, i}} q_{y s, i}(x) \mathrm{d} x+\int_{x_{s t, i}}^{a_{i}} q_{y t, i}(x) \mathrm{d} x,
$$

where $q_{y s, i}(x)$ is the traction force at point $x$ in the sliding region along the tire's ego direction, and $q_{y t, i}(x)$ is the traction force at point $x$ in the adhesive region along the tire's ego direction.

Modeling the brush elements as linear springs with stiffness $c_{y, i}$, the lateral displacement of the brushes in the adhesive region is proportional to the tangent of the slip angle. This lateral displacement produce the force

$$
q_{y t, i}(x)=c_{y, i}\left(a_{i}-x\right) \tan \left(\alpha_{i}\right) .
$$

Assuming a parabolic distribution of the total vertical load, gives at each point $x$ along the contact patch the vertical load

$$
q_{z, i}(x)=\frac{3}{4} F_{z, i} \frac{a_{i}^{2}-x^{2}}{a_{i}^{3}} .
$$


For a given friction coefficient $\mu_{y, i}$, the sliding force at each point along the contact patch is

$$
q_{y s, i}(x)=\operatorname{sign}\left(\alpha_{i}\right) \mu_{y, i} q_{z, i}(x) .
$$

The thread elements start sliding once the force produced by their displacements exceed the static friction keeping them fixed to the road. To compute the point $x_{s t, i}$ where the thread elements start sliding, it is checked when $q_{y s, i}$ is equal to $q_{y t, i}$ :

$$
q_{y t, i}\left(x_{s t, i}\right)=q_{y s, i}\left(x_{s t, i}\right) .
$$

This gives a solution at $x_{s t, i}=a_{i}$ where the vertical load $q_{z, i}=0$, but the interesting solution where the thread elements start sliding is given by

$$
x_{s t, i}=\frac{4 c_{y, i} a_{i}^{3} \tan \left(\alpha_{i}\right)}{3 \mu_{y, i} F_{z, i}}-a_{i} .
$$

For small slip angles, where as $\alpha_{i} \rightarrow 0$ then $x_{s t, i} \rightarrow-a_{i}$ and $\tan \left(\alpha_{i}\right) \rightarrow$ $\alpha_{i}$, the lateral force resulting from $(2.43)$ is

$$
F_{y 0, i}=\int_{-a_{i}}^{a_{i}} q_{y t, i}(x) \mathrm{d} x=\int_{-a_{i}}^{a_{i}} c_{y, i}\left(a_{i}-x\right) \alpha_{i} \mathrm{~d} x=c_{y, i} a^{2} \alpha_{i} .
$$

This correspond to the often used linear tire model

$$
F_{y 0, i}=C_{\alpha, i} \alpha_{i},
$$

where $C_{\alpha, i}=c_{y, i} a_{i}^{2}$ is the lateral tire stiffness. By applying this linear tire model to the single-track model in Section 2.2.3, using small-angle approximations, and assuming the longitudinal velocity to be constant, this results in a linear model of the vehicle lateral dynamics. This has the obvious benefit of enabling linear control synthesis such as the linear quadratic regulator (LQR) [60], and can be used for model predictive control (MPC) with performance guarantees [61].

Solving (2.43) without assuming small slip angles gives the Fiala brush model [62]:

$$
\begin{aligned}
F_{y 0, i} & =\left\{\begin{array}{cc}
C_{\alpha, i} \tan \left(\alpha_{i}\right) \\
-\frac{C_{\alpha, i}^{2}\left|\tan \left(\alpha_{i}\right)\right| \tan \left(\alpha_{i}\right)}{3 \mu_{y, i} F_{z, i}} \\
+\frac{C_{\alpha, i}^{3} \tan ^{3}\left(\alpha_{i}\right)}{27 \mu_{y, i}^{2} F_{z, i}^{2}}, & \text { if }\left|\alpha_{i}\right|<\alpha_{\mathrm{sl}}, \\
\mu_{y, i} F_{z, i} \operatorname{sign}\left(\alpha_{i}\right), & \text { otherwise }
\end{array}\right. \\
\alpha_{\mathrm{sl}} & =\arctan \left(3 \mu_{y, i} F_{z, i} / C_{\alpha, i}\right),
\end{aligned}
$$


where $\alpha_{\mathrm{sl}}$ is the slip angle where total sliding occurs $\left(x_{s t, i}=a_{i}\right)$. Similar expressions based on the brush model can also be derived for the longitudinal tire force [59]. The brush model is used in Paper IV. The model is suitable when driving up to the saturation limits of the tires, such as path tracking at the limit of friction [63] and nonlinear MPC for collision avoidance [30]. The brush model is physically motivated, models the saturation behavior of the tire forces, and only contains one extra parameter when compared to the linear tire model (2.50). It is, however, not as accurate when compared to measurement data as the Pacejka's Magic Formula tire model [58], which is described in Section 2.3.4. The brush models have many variations and extensions to improve their accuracy, but this comes at a cost in terms of an increased number of parameters. One addition is to use separate friction coefficients to model the adhesive friction and the sliding friction [64]. This can be further detailed by modeling the fact that the sliding friction depends on the sliding velocity [65]. With its roots in physical modeling, the brush model provides some insights into the tire traction forces. For further analytical insights of the tire behavior, unsteady-state versions of the brush model can be considered [66].

\subsubsection{Magic Formula Tire Model}

The Pacejka's Magic Formula model is a so called semi-empirical tire model, which provides a parameterization of the steady-state tire traction forces. The longitudinal forces $F_{x 0, i}$ and the lateral forces $F_{y 0, i}$ of the tires are modeled using Pacejka's Magic Formula [58] and are given by

$$
\begin{aligned}
F_{x 0, i}= & D_{x, i} \sin \left(C _ { x , i } \operatorname { a r c t a n } \left(B_{x, i} \kappa_{i}\right.\right. \\
& \left.-E_{x, i}\left(B_{x, i} \kappa_{i}-\arctan \left(B_{x, i} \kappa_{i}\right)\right)\right), \\
F_{y 0, i}= & D_{y, i} \sin \left(C _ { y , i } \operatorname { a r c t a n } \left(B_{y, i} \alpha_{i}\right.\right. \\
& \left.\left.-E_{y, i}\left(B_{y, i} \alpha_{i}-\arctan \left(B_{y, i} \alpha_{i}\right)\right)\right)\right),
\end{aligned}
$$

where $B_{x, i}, C_{x, i}, D_{x, i}, E_{x, i}, B_{y, i}, C_{y, i}, D_{y, i}$, and $E_{y, i}$ are model parameters. A comparison of the Pacejka's Magic Formula, the Fiala brush model, and the linear tire model is shown in Figure 2.10. In this thesis, it is assumed that (2.52a) and (2.52b) scale linearly with the tire-road friction coefficients and longitudinal force by replacing $D_{x, i}$ and $D_{y, i}$ with

$$
\begin{aligned}
D_{x, i} & =\mu_{x, i} F_{z, i}, \\
D_{y, i} & =\mu_{y, i} F_{z, i} .
\end{aligned}
$$




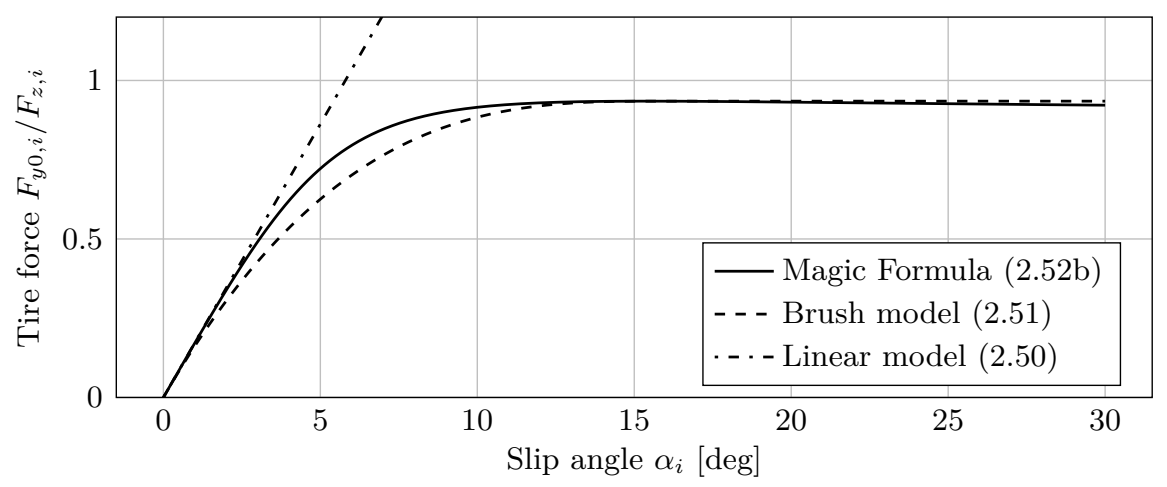

Figure 2.10: Comparison between lateral tire models.

In reality, changed vertical load influences the tire-road friction making the relation nonlinear, where the load-transfer when cornering leads to a total reduction of the cornering-force capability for tires on the same axle [39]. The assumption that the tire forces are linear in the road friction coefficient causes the tire cornering stiffness to change with road friction. This is not in correspondence with real tire behavior where the cornering stiffness is typically constant as it is dependent on tire forces due to adhesion, which as described in Section 2.3.3 is a property of the tire deformation rather than the sliding friction between tire and road [58]. Formulas for scaling of the parameters in (2.52a) and (2.52b) for changing vertical loads and friction conditions can be found in [58,67], which are based on experimental data.

The Pacejka's Magic Formula is used for various purposes in all papers included in this thesis. Because of its ability to fit well with measurement data, its use is widespread. Some examples of its use are examining how control strategies differ for different road conditions [68], friction estimation [69], nonlinear MPC [70], and post-impact vehicle stabilization [71].

\subsubsection{Combined Slip}

This section looks at tire forces under combined slip conditions on the form

$$
\begin{aligned}
& F_{x, i}\left(\kappa_{i}, \alpha_{i}\right), \\
& F_{y, i}\left(\kappa_{i}, \alpha_{i}\right),
\end{aligned}
$$


where the influence of both the longitudinal and lateral slips on both the longitudinal and lateral tire forces are considered. It is experimentally challenging to collect data for combined-slip conditions, thus it is common to extend the pure-slip models described in the previous sections to the combined slip case. One option to do this is to scale the original forces with a weighting function that depends on the combined slip, i.e.,

$$
\begin{aligned}
& F_{x, i}\left(\kappa_{i}, \alpha_{i}\right)=G_{x, i}\left(\kappa_{i}, \alpha_{i}\right) F_{x 0, i}\left(\kappa_{i}\right), \\
& F_{y, i}\left(\kappa_{i}, \alpha_{i}\right)=G_{y, i}\left(\kappa_{i}, \alpha_{i}\right) F_{y 0, i}\left(\alpha_{i}\right) .
\end{aligned}
$$

Examples of such weighting functions are those used with the Magic Formula [58]:

$$
\begin{aligned}
H_{x \alpha, i} & =B_{x 1, i} \cos \left(\arctan \left(B_{x 2, i} \kappa_{i}\right)\right), \\
G_{x, i} & =\cos \left(C_{x \alpha, i} \arctan \left(H_{x \alpha, i} \alpha_{i}\right)\right), \\
F_{x, i} & =G_{x, i} F_{x 0, i}, \\
H_{y \kappa, i} & =B_{y 1, i} \cos \left(\arctan \left(B_{y 2, i} \alpha_{i}\right)\right), \\
G_{y, i} & =\cos \left(C_{y \kappa, i} \arctan \left(H_{y \kappa, i} \kappa_{i}\right)\right), \\
F_{y, i} & =G_{y, i} F_{y 0, i},
\end{aligned}
$$

where $B_{x 1, i}, B_{x 2, i}, C_{x \alpha, i}, B_{y 1, i}, B_{y 2, i}$, and $C_{x \kappa, i}$ are model parameters. An example of the resultant force given with this model for different combinations of tire slips is shown in Figure 2.11. This tire model of combined slip is used together with Pacejka's Magic Formula in all papers included with this thesis.

A simpler model for combined slip is the friction-ellipse based combined-slip model [39]. It is based around extending the fact that the total tire forces are limited by an elliptic relation

$$
\left(\frac{F_{x, i}}{F_{x 0, i}^{\max }}\right)^{2}+\left(\frac{F_{y, i}}{F_{y 0, i}^{\max }}\right)^{2} \leq 1,
$$

where $F_{x 0, i}^{\max }=\mu_{x, i} F_{z, i}$ and $F_{y 0, i}^{\max }=\mu_{y, i} F_{z, i}$ are the maximum achievable longitudinal and lateral tire forces, respectively. If the total tire force is saturated, then (2.56) gives that

$$
F_{y, i}=F_{y 0, i}^{\max } \sqrt{1-\left(\frac{F_{x, i}}{F_{x 0, i}^{\max }}\right)^{2}} .
$$

This can be taken advantage of to model the driving behavior when the tire forces are fully saturated, such as for drifting maneuvers [72]. In the 


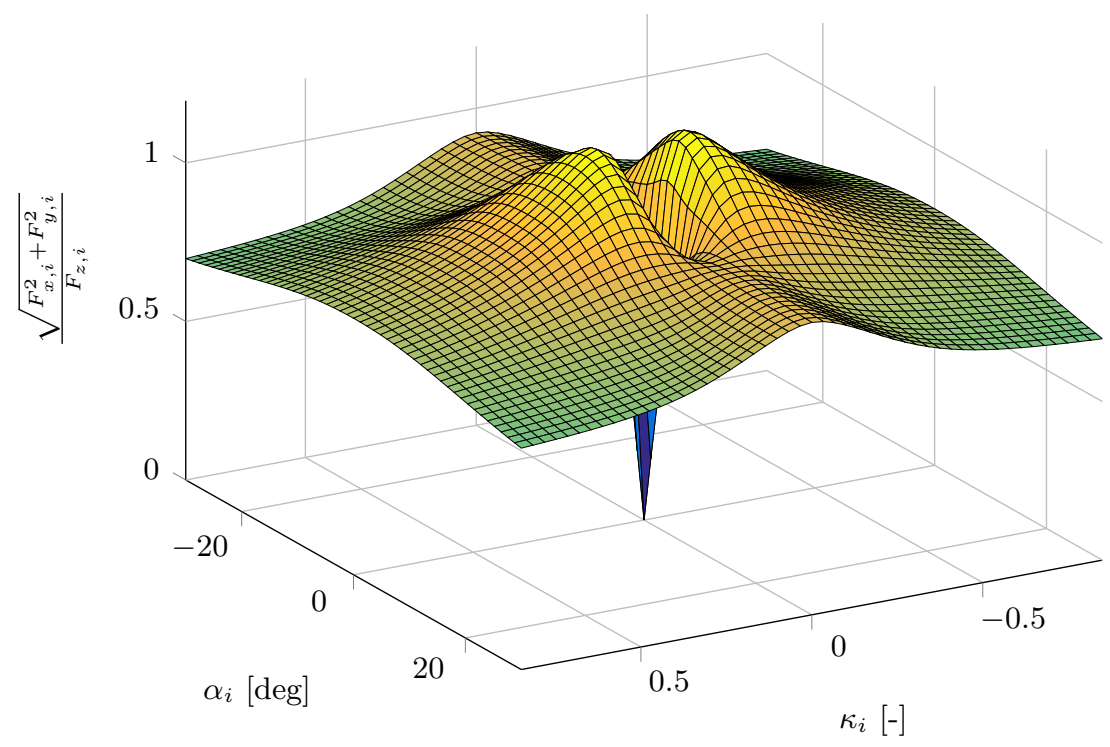

Figure 2.11: Force-slip diagram of Pacejka's Magic Formula with weighting functions using parameters corresponding to dry asphalt interaction. The resultant force is normalized with the normal force.

friction-ellipse based model, this is assumed to also hold for any nominal lateral tire force [59]

$$
F_{y, i}=F_{y 0, i} \sqrt{1-\left(\frac{F_{x, i}}{F_{x 0, i}^{\max }}\right)^{2}} .
$$

When using this model, the longitudinal force $F_{x, i}$ is typically directly considered a vehicle input, which is reasonable if disregarding the wheel dynamics (2.35) such that the braking force is directly achievable via selection of the braking torque $T_{i}=R_{e} F_{x, i}$. A comparison between the frictionellipse based model and weighting functions is shown in Figure 2.12. The friction-ellipse based combined-slip model is used to determine appropriate braking signals in Papers III, IV, and VI.

\subsection{Discussion}

Depending on the intended usage of a vehicle model, there are different requirements and preferences. It is important that the vehicle model used is able to handle important phenomena relevant for its intended use. In 


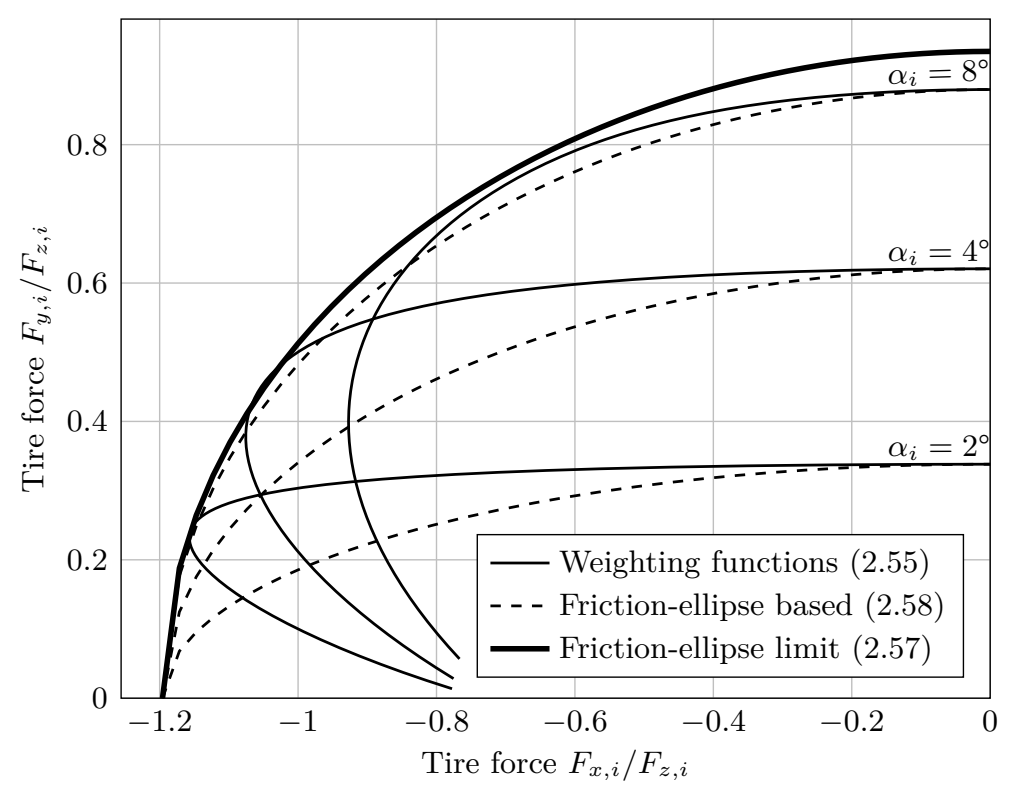

Figure 2.12: Comparison between the different combined-slip models.

simulation, edge cases of models are seldom explored and accurate models such as those available in CarMaker [25] that behave very similar to a real car are useful. An accurate model can be more convincing when evaluating a controller implementation than when using an idealized model close to that used to develop the controller. In particular, it can demonstrate the controller's robustness to model uncertainties.

For optimal control, such complexity can instead result in unrealistic trajectories dependent on detailed intrinsics of the model where nonphysical behavior $[20,73]$ is exploited. It can also be desirable for the model to not be overly specified, in order for the results to not be more dependent on specific vehicle parameters than necessary and remain representative for the general behavior of a wider range of vehicles. The characteristics of optimal maneuvers can be highly dependent on the road condition and the vehicle model used, see [68] and [73], respectively. Additionally, the algorithms required to effectively solve the resulting optimization problem can be reliant on the model being twice differentiable with numerically well behaved Jacobian and Hessian. Tire models containing normalized terms $\left([x, y]^{\mathrm{T}} / \sqrt{x^{2}+y^{2}}\right)$ of tire slips, to model combined tire forces, can for example experience problems in numerical optimization algorithms because of the singularity in the Jacobian when 

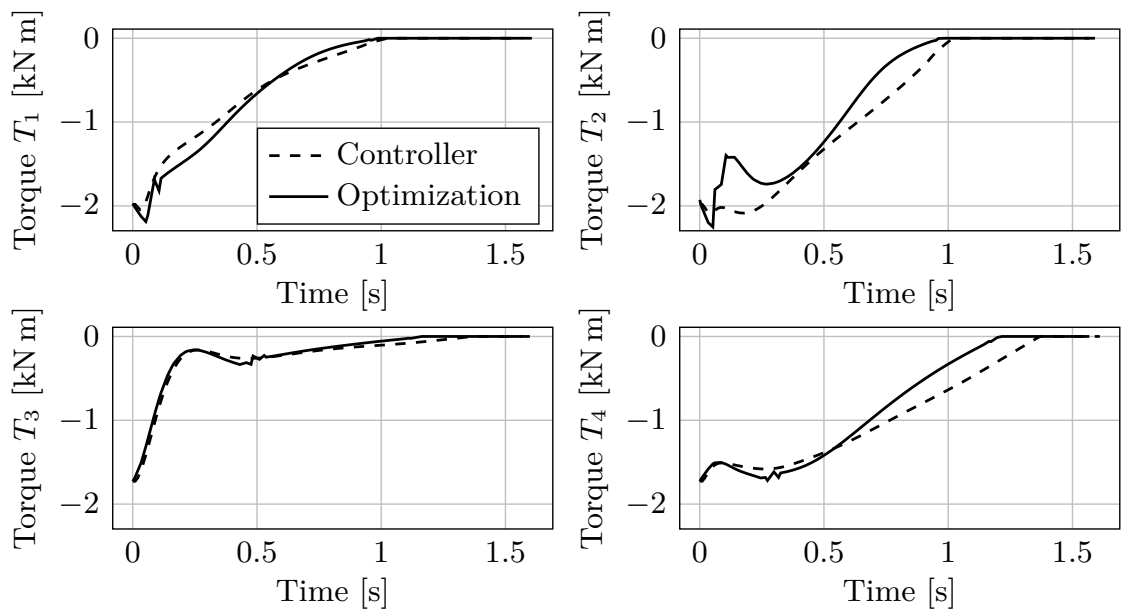

Figure 2.13: Comparison of braking torques for offline numerical optimization using the Pacejka's Magic Formula tire model with weighting functions (2.55) and the controller (CL FE) developed in Paper III simulated on the same model but that internally uses the friction-ellipse based model (2.58).

one of the terms is zero.

For the application of numerical optimal control, this thesis makes extensive use of the vehicle model referred to as DT WF by [73]. In this model, the weighting functions (2.55) are used to model combined slip, which does not have the potential problem of singularities in the Jacobian discussed earlier. The name of the vehicle model used, DT WF, comes from that it is a double-track model using Pacejka's Magic Formula (2.52) with weighting functions (2.55) to model combined longitudinal and lateral tire forces. The DT WF model also models the pitch and yaw dynamics as described in Section 2.2.5. The different vehicle model parameters used in the thesis are collected in Appendix A.

For the application of computing appropriate braking for online control, the friction-ellipse based combined-slip model (2.58) is used in this thesis. When the developed controller (CL FE) in Paper III is simulated with the DT WF model, the resulting braking torques are very similar to those obtained using numerical optimization with the DT WF model. Figure 2.13 shows the resulting braking torques for the scenario from $\mathrm{Pa}-$ per III, where a vehicle enters a curve with curve radius $50 \mathrm{~m}$ at $90 \mathrm{~km} / \mathrm{h}$ and has to steer and brake to minimize its deviation from the road centerline. Even though the models used to compute the braking torques differ (see Figure 2.12), the resulting braking torques are remarkably similar. 



\section{Chapter 3}

\section{Conclusion}

$\mathrm{T}$ o minimize the risk of collision, the first step is to plan the vehicle motion. The vehicle motion is determined by a trade-off between controlling the longitudinal force, the lateral force, and the yaw moment acting on the vehicle. These are determined by the sum of the individual tire forces acting on the four wheels. In turn, these are limited by, e.g., the tire-road friction and wheel load, and are determined by the tire slips resulting from the vehicle motion, the steering angle, and the wheel speeds. Out of these, the steering angle and wheel speeds can be directly controlled by steering and applying wheel torques. With a force-centric perspective, this research examines how to efficiently control the steering wheel and the brakes, given a detected safety-critical scenario such as collision avoidance. In this chapter, a summary of the contributions in the thesis is given for each paper and a reflection on the research is made along with an outlook on areas for further exploration.

\subsection{Contributions}

\section{Paper I}

V. Fors, B. Olofsson, and L. Nielsen, "Formulation and interpretation of optimal braking and steering patterns towards autonomous safetycritical manoeuvres," Vehicle System Dynamics, vol. 57, no. 8, pp. 1206-1223, 2019.

In the first two papers, numerical optimization is used to examine the optimal behavior of a vehicle in safety-critical scenarios. A formulation 

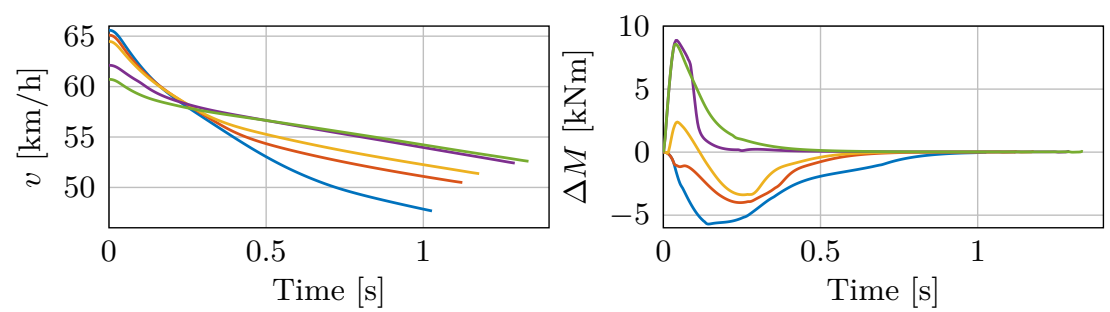

Figure 3.1: Velocity $v$ and brake-induced yaw moment $\Delta M$ during a lefthand turn maneuver for different trade-offs between the initial and final velocity. Adapted from Paper I.

is introduced with an optimization criterion using a single interpolation parameter $\eta$ to form a linear combination of the initial and final velocity of the maneuver. This formulation results in a family of braking and steering patterns for vehicles in at-the-limit maneuvers, highlighting the optimal behavior for different levels of braking in critical scenarios (see Figure 3.1). Heavy braking of the inner wheels when turning is observed for all values of the interpolation parameter $\eta$, while increased weight to the final velocity leads to a reduced braking effort on the outer wheels. This hints at future lane-keeping control systems that can seamlessly adapt the level of braking depending on the situation at hand, and at the boundary values for the parameter $\eta$ the two different strategies of optimal lane-keeping control and optimal yaw control are embedded. It is observed that for optimal lane-keeping control, the individual tire forces are close to their friction limits. The steering and braking patterns show significant similarities in behavior for different scenarios, suggesting a single control framework could handle a multitude of situations. Using the optimization as a benchmark, a constant slip-angle strategy is shown to accomplish close to that of optimal steering for different levels of braking.

\section{Paper II}

V. Fors, B. Olofsson, and L. Nielsen, "Attainable force volumes of optimal autonomous at-the-limit vehicle manoeuvres," Vehicle System Dynamics, vol. 58, no. 7, pp. 1101-1122, 2020.

The attainable forces of optimal autonomous maneuvers are examined and compared to the actual force utilization in optimal vehicle maneuvers. A new illustration named attainable force volumes is introduced (see Figure 3.2), and it is used to effectively show how the trajectory of a vehicle maneuver relates to the attainable forces over the duration of 


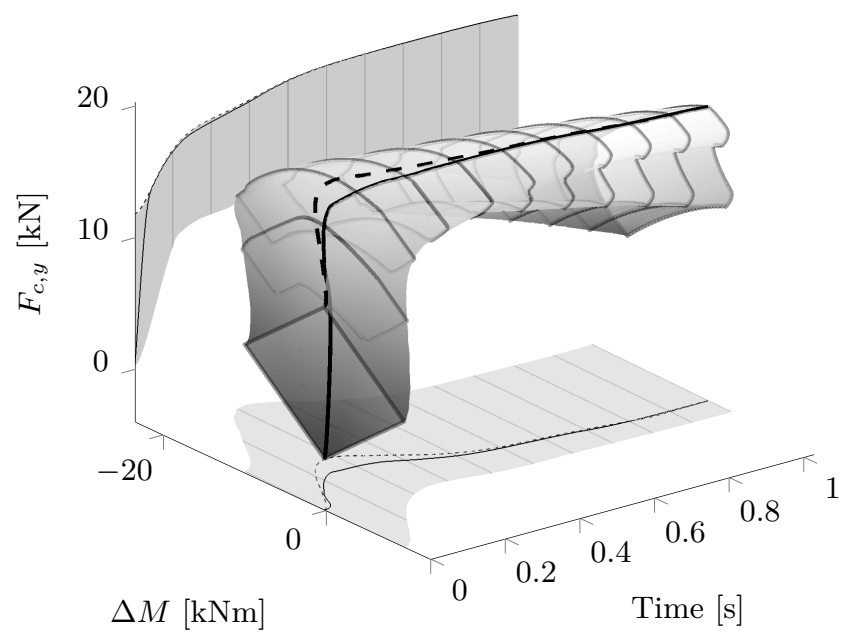

Figure 3.2: Attainable force volume during a left-hand turn. The volume contains the attainable forces from braking with the optimal trajectory shown as a solid line. Adapted from Paper II.

the maneuver. To enable this illustration, the globally fixed control-force vector $F_{c}$ is defined and justified using Pontryagin's maximum principle on a simplified vehicle model. For optimal lane keeping, the trajectories are after an initial transient observed to develop on the boundary surface of the attainable force volume spanned by the control-force vector component $F_{c, y}$ and the yaw moment $\Delta M$. Applied to lane-keeping control, this indicates a set of control principles similar to those analytically obtained for friction-limited particle models in earlier research, but is shown to result in vehicle behavior close to the globally optimal solution also for more general models and scenarios. Specifically, it is important to maximize the force in a control-force component, rather than to control the direction of the force vector. Further, from the attainable force volumes it is found in both a left-hand turn scenario and the initial turn of a double lane-change scenario that the trajectories are close to their maximum attainable control-force component $F_{c, y}$. This means that direct yaw-moment control is not prioritized over maximizing the control-force component $F_{c, y}$, which allows for simplifications in control design. 

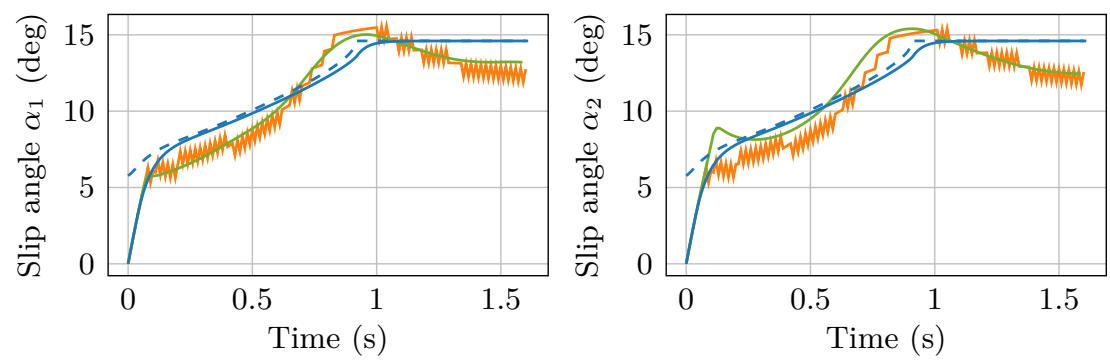

Figure 3.3: Front-wheel slip angles obtained for the developed controller (CL FE) in blue with desired values as dashed, the alternative steering controller [77] in orange, and the offline numerical optimization in green. Adapted from Paper III, Copyright (C) 2019 IFAC.

\section{Paper III}

V. Fors, B. Olofsson, and L. Nielsen, "Yaw-moment control at-the-limit of friction using individual front-wheel steering and four-wheel braking," in Proceedings of the 9th IFAC International Symposium on Advances in Automotive Control (AAC), vol. 52, no. 5, pp. 458-464, Orléans, France, 2019.

A controller for operation at the limit of friction using front-wheel steering and individual four-wheel braking is developed based on following an acceleration-vector reference. By exploiting the finding in Paper I that the individual tire forces when driving under optimal lane keeping tend to be close to their friction limits, the desired tire slip angles are computed given an acceleration reference. This enables the use of proportional feedback controllers to achieve steering and body-slip control. A new simplified combined-slip tire model that only considers the extreme case at the friction limit is introduced. Using this model to compute the desired slip angles, and using a friction-ellipse based model to compute the desired braking forces, the steps to compute the steering and braking inputs from an acceleration reference of the vehicle center of mass are explicit, resulting in a computationally efficient controller. To enable different acceleration-vector references for each tire, the controller is developed under the assumption that the front wheels are individually steered, making the control input for each tire independent. A simple adaptation of the developed controller is demonstrated to enable it to be used for a more conventional vehicle with a single steering input. The controller is evaluated on a lane-departure prevention problem us- 
ing a friction-limited particle model to compute the acceleration-vector reference. When compared with offline numerical optimization and an alternative steering controller based on a local minimization strategy [77], it is found that while the resulting steering angles differ, the slip angles of the front wheels between these methods and the developed controller are very similar (see Figure 3.3). The resulting control performance of the developed controller is comparable to that of the much more computationally demanding offline numerical optimizations. Compared to the alternative steering controller [77], the performance of the developed controller is similar, but thanks to proportional feedback the developed controller provides a smoother steering trajectory.

\section{Paper IV}

\section{Fors, B. Olofsson, and L. Nielsen, "Autonomous wary collision avoidance," IEEE Transactions on Intelligent Vehicles, 2020, DOI 10.1109/TIV.2020.3029853.}

When avoiding an obstacle by straight-line braking, ABS can intervene to provide close to optimal braking without any prior information about the road friction. Inspired by this, a controller for autonomous collision avoidance that does not use any estimation of the tire-road friction is developed. The developed controller is based on a wary strategy to perform the maneuver that requires the minimum tire-road friction. Using the available actuation according to the least friction for which collision is still avoidable, results in a computation of the controller output that is independent of the actual tire-road friction. The controller is based on

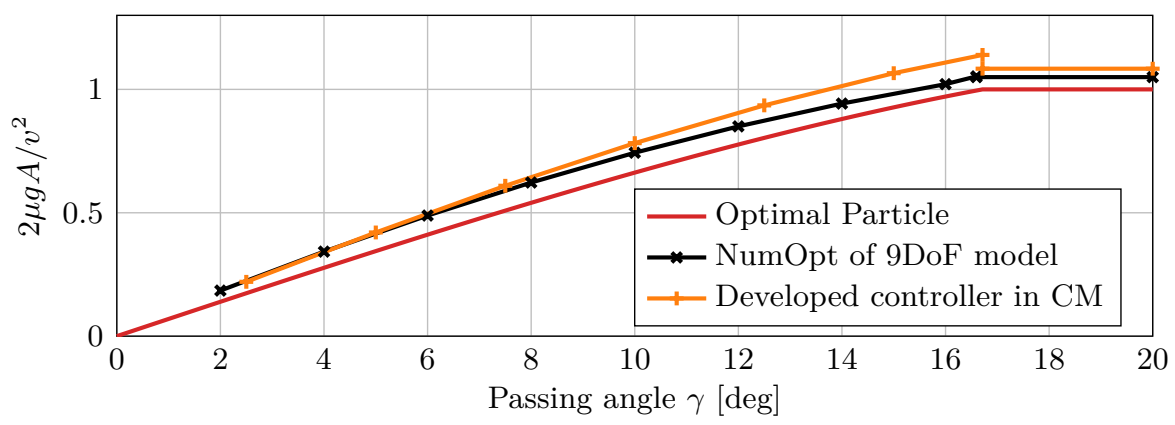

Figure 3.4: Performance comparison between different models and controllers at different initial passing angles $\gamma$. Lower is better. Adapted from Paper IV, Copyright (C) 2020 IEEE. 
the controller in Paper III, but rather than using the empirical frictionlimit tire model introduced in that paper, an analytical expression for the relation between the friction limit and the slip angle is derived. Like in Paper III, the acceleration reference for the vehicle center of mass is computed using a friction-limited particle model, which in the case of the wary strategy has an explicit analytical solution, resulting in the controller being fully explicit. The applicability of this analytical solution is verified by numerical optimization on a full vehicle model using the concepts of the control force and attainable force volume from Paper II. Despite the low computational requirements of the developed controller and not needing information about the actual tire-road friction, it is demonstrated to be able to handle situations close to that achieved by offline numerical optimization (Figure 3.4).

\section{Paper V}

V. Fors, Y. Gao, B. Olofsson, T. J. Gordon, and L. Nielsen, "Real-time minimum-time lane change using the modified Hamiltonian algorithm," in Proceedings of the 26th Symposium of the International Association of Vehicle System Dynamics (IAVSD), pp. 1457-1465, Gothenburg, Sweden, 2019.

Aiming to perform real-time minimum-time lane changes, the Modified Hamiltonian Algorithm (MHA) [77] (see Appendix B) is adapted. In a

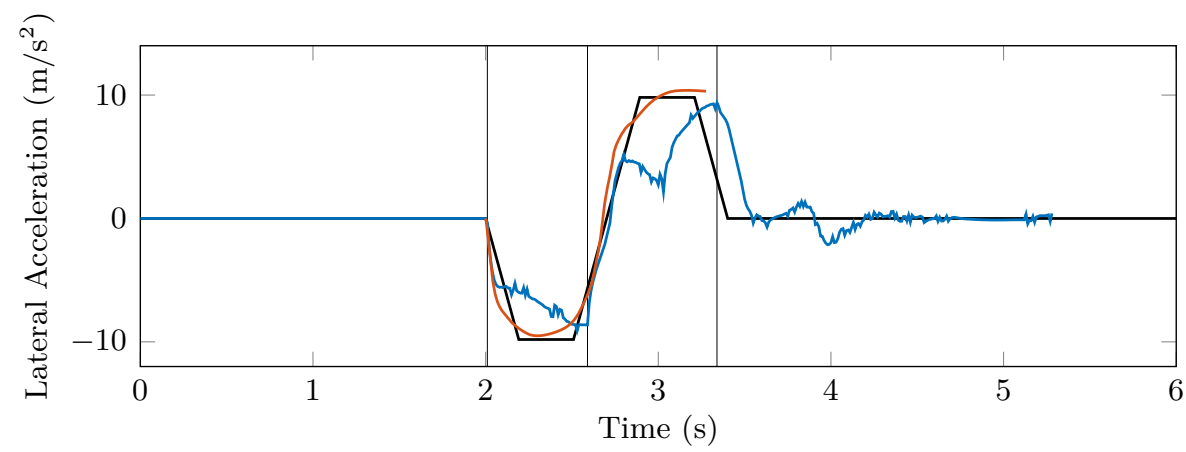

Figure 3.5: Path lateral acceleration for the optimal particle (black), the adapted MHA method (blue), and offline numerical optimization (red). The four different phases of the MHA controller: constant velocity, accelerate to the right, accelerate to the left, and stabilize while braking are separated with vertical lines. Adapted from Paper V, Copyright @ 2020 Springer Nature Switzerland AG. 
minimum-time lane change a key variable is the switching time of the acceleration reference, i.e., when to stop the lateral acceleration towards the new lane and instead decelerate the lateral motion to stabilize at the correct new lane position. It is shown that the simple friction-limited particle model used in Papers III-IV is insufficient for this scenario, leading to an overshoot of the desired lane position. By incorporating a limitedjerk condition in the particle model to account for the yaw dynamics of the vehicle, the switching time can successfully be predicted (see Figure 3.5). Further, a stabilizing modification of the acceleration reference is introduced for the vehicle to converge to the desired lane position.

\section{Paper VI}

\section{Fors, P. Anistratov, B. Olofsson, and L. Nielsen, "Predictive force- centric emergency collision avoidance by steering and individual four- wheel braking," 2020, Manuscript.}

A controller for critical vehicle maneuvering is proposed that achieves heavy braking while avoiding obstacles and staying on the road. The desired motion of the center of mass is planned by a receding-horizon approach, where the computed acceleration profile is followed by adapting the controller from Paper III. The motion-planning step uses a frictionlimited particle model with elliptic friction constraints to capture the difference in friction in longitudinal and lateral directions. Compared to the analytically obtained acceleration-vector references in Papers IV-V,

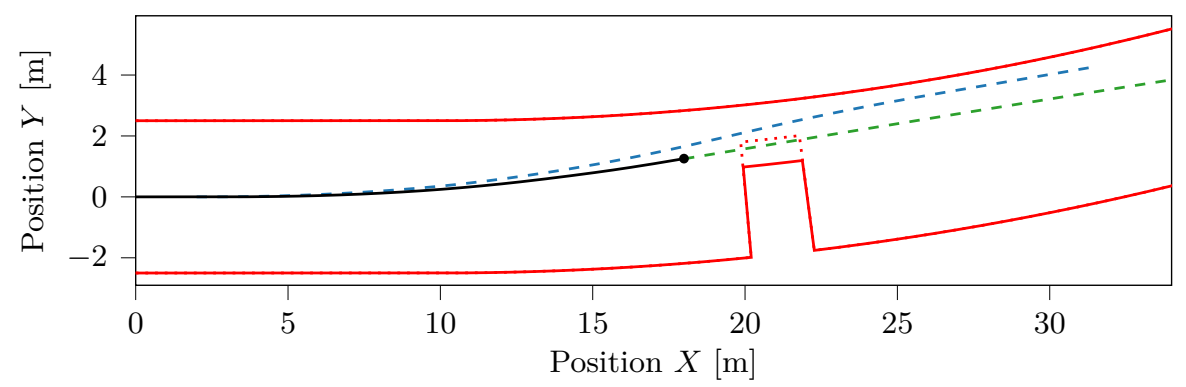

Figure 3.6: The path for a scenario featuring a moving obstacle in a curved road. The red lines mark the position constraints with the dotted red line marking the obstacle constraint at the start of the maneuver. The small black dot is the current position of the car with a black line showing its path. The dashed blue line is the first planned path. The dashed green line is the latest planned path. Adapted from Paper VI. 
the receding-horizon approach enables a more general scenario definition and more freedom to choose the optimization criterion. The controller is evaluated in a number of representative scenarios where it demonstrates real-time performance. In all cases, the vehicle is well behaved regarding steering angles, slip angles, body slip, and the resulting paths. By replanning, the controller takes advantage of braking possibilities when the obstacle moves to widen the drivable area (see Figure 3.6). In conclusion, the controller successfully balances between braking and avoidance, performing well both from a functional perspective and from a real-time perspective.

\section{Other Publications by the Author}

V. Fors, B. Olofsson, and L. Nielsen, "Formulation and interpretation of optimal braking patterns in autonomous lane-keeping maneuvers," presented at the 2nd IAVSD Workshop on Dynamics of Road Vehicles, Berlin, Germany, 2017.

Initial research presented at a workshop that led to the Papers I-II.

\section{Fors, B. Olofsson, and L. Nielsen, "Slip-angle feedback control for autonomous safety-critical maneuvers at-the-limit of friction," pre- sented at the 14th International Symposium on Advanced Vehicle Con- trol (AVEC), Beijing, China, 2018. URL: http://urn.kb.se/resolve?urn= urn:nbn:se:liu:diva-149899.}

Initial research presented at a conference that led to the Papers III-IV. This research was limited in scope by not considering the yaw stability of the vehicle.

\subsection{Reflection}

This research has investigated autonomous safety-critical maneuvering of passenger cars. The research started with a study of the optimal behavior, with an ambition to provide insights that can be used to aid in design of future safety systems. Autonomous safety systems inspired by these results have the potential to save lives [83]. The application of the insights gained when studying the optimal behavior was particularly suitable for methods based on following acceleration-vector references [71, 77]. Further, the results in Paper II directly hint at the close-to-optimal potential of such methods, which have been the focus of the later research. 
The goal of the acceleration-based methods is to first find an acceleration-vector reference for the given scenario, then given this reference find appropriate steering and braking commands. With the individual tire forces summing up to the total force and the total moment acting on the vehicle, and the desire to utilize all available tire forces, a procedure to efficiently compute the slip angle and braking torque for which the tire force is maximized in a given direction is valuable. The methods introduced in Papers III-IV are appealing approaches to achieve this.

A particular advantage of the acceleration-based methods investigated in this thesis is that they allow the usage of different models to compute the steering and the braking actions, while still taking combined steering and braking into consideration. In Papers III-IV, the tire models are tailored for their specific use (such as only describing the behavior at the friction-ellipse limit) which enable them to remain simple while still working well for their respective use case. An example of this can be observed in Figures 3.3 and 2.13, where different tire models are used to determine the steering and braking, respectively, both models resulting in behavior close to that obtained by offline numerical optimization.

Computing the desired slip angles given a desired acceleration vector gives some intuition on the optimal behavior. A special case is the constant front-axle slip-angle strategy inspired by professional racing drivers [84], which is shown to be close to optimal in a left-hand turn scenario in Paper I. In that case, the desired acceleration is close to perpendicular to the velocity vector of the vehicle, resulting in the optimal slip angles being close to those that provide maximum lateral force.

A goal stated in Section 1.2 is to find control principles that allow for simplifications in the problems of planning, control, and parameter estimation when operating at the limit of friction. The culmination of this effort is Paper IV, where a controller is developed that performs close to that achieved by offline numerical optimization, while being independent of any estimation of the tire-road friction and being fully explicit, making its computational time negligible. A takeaway is that like one may make simplifications in the control design when driving under mild conditions, one may make other simplifications when driving at the limit.

\subsection{Outlook}

While the findings in Paper I indicate future safety systems that can adapt the level of braking depending on the situation at hand, interpolat- 
ing between yaw-moment control and optimal lane-keeping control, this is not explored further in this thesis. The wary controller developed in $\mathrm{Pa}-$ per IV has a similar concept, where the least amount of friction for which avoidance is still possible is used to determine the steering and braking actions, but with this determination of braking and with full authority over the steering, the resulting strategy is different. On reflection, the kind of adaption suggested in Paper I appears more suitable for driver-assistance functions, where it may be desirable to give the driver more control and limit the intervention. Driver-assistance functions that support the driver and include a driver in the loop have not been considered in this thesis, in favor of autonomous functions with full authority over the steering and braking. A driver-assistance system with different degrees of intervention is demonstrated in [85], but there the different degrees of intervention are discrete rather than the continuous scale suggested in Paper I.

The research in this thesis is focused on passenger cars. The possibility of rollover introduces another constraint on the system, where the lateral acceleration has to be limited to prevent the vehicle from tipping over. This is highly relevant for larger vehicles such as trucks, but also needs to be considered for passenger cars with higher center of gravity such as SUVs. Here, the acceleration-based methods developed could be adapted to take rollover into account when planning and following the desired acceleration.

An alternative approach to coordinate the steering and braking with the vehicle motion is to use MPC on a full vehicle model where the problems of motion planning and control are solved simultaneously [30, 86]. The methods and models for the acceleration-based controllers developed in this theses can potentially be of use also for this architecture. In particular, the analytical model of the tire-road friction limit in Paper IV could be used for the prediction or to achieve the tire forces predicted.

Robustness to friction is achieved in Paper IV, where it is eliminated as an estimated variable from the control loop. From the perspective of safety, it would be interesting to further study the methods' ability to handle uncertainties in parameters and realistic disturbances, and to further develop the methods to increase such robustness.

The controllers developed in this thesis show great promise with low computational cost and performance not far from that obtained offline by using numerical optimization when evaluated in high-fidelity simulation, and it is thus interesting how these results transfer to experiments on real vehicles. 


\section{Appendix A}

\section{Vehicle Model Parameters}

$\mathrm{T}$ HE parameters used to model the vehicle in Papers I, II, III, and VI are collected in Table A.1. These parameters have been used by [87], where the tire parameters originate from the empirical model presented by [58]. They are chosen to resemble the dynamics of a standard passenger car on dry asphalt. Not all parameters are used in every paper, which parameters that are used depend on the exact modeling equations used in that paper. For example, Paper VI uses static load transfer and thus does not use the parameters related to the suspension system.

Paper IV and V use different parameters that try to resemble the high-fidelity CarMaker [25] model used for simulation. These parameters are similar to those for a Ford Focus and are collected in Table A.2. The same suspension parameters as previously are used and the other chassis parameters are obtained from the model description in CarMaker. The tire parameters have been fitted based on slip-force curves for different combinations of slip ratios and slip angles under nominal tire loads, corresponding to the vehicle at zero longitudinal and lateral acceleration. 
Table A.1: Vehicle model parameters used in Papers I, II, III, and VI. The chassis model parameters are from [87] and resemble the dynamics of a standard passenger car. The tire-force model parameters originate from [58], representing the behavior of a tire on dry asphalt.

\begin{tabular}{|c|c|c|c|}
\hline Description & Notation & Value & Unit \\
\hline \multirow{2}{*}{ Longitudinal distance to wheel $i$} & $l_{x, 1}, l_{x, 2}$ & 1.3 & $\mathrm{~m}$ \\
\hline & $l_{x, 3}, l_{x, 4}$ & -1.5 & $\mathrm{~m}$ \\
\hline \multirow{2}{*}{ Lateral distance to wheel $i$} & $l_{y, 1}, l_{y, 3}$ & 0.8 & $\mathrm{~m}$ \\
\hline & $l_{y, 2}, l_{y, 4}$ & -0.8 & $\mathrm{~m}$ \\
\hline Height of the center of mass & $h$ & 0.5 & $\mathrm{~m}$ \\
\hline Vehicle mass & $m$ & 2100 & $\mathrm{~kg}$ \\
\hline Roll moment of inertia & $I_{x x}$ & 765 & $\mathrm{~kg} \mathrm{~m}^{2}$ \\
\hline Pitch moment of inertia & $I_{y y}$ & 3477 & $\mathrm{~kg} \mathrm{~m}^{2}$ \\
\hline Yaw moment of inertia & $I_{z z}$ & 3900 & $\mathrm{~kg} \mathrm{~m}^{2}$ \\
\hline Pitch suspension stiffness & $K_{\theta}$ & 363540 & $\mathrm{Nm} / \mathrm{rad}$ \\
\hline Pitch suspension damping & $D_{\theta}$ & 30960 & $\mathrm{Nms} / \mathrm{rad}$ \\
\hline Roll suspension stiffness & $K_{\phi}$ & 178000 & $\mathrm{Nm} / \mathrm{rad}$ \\
\hline Roll suspension damping & $D_{\phi}$ & 16000 & $\mathrm{Nms} / \mathrm{rad}$ \\
\hline Rotational wheel inertia & $I_{w}$ & 2 & $\mathrm{~kg} \mathrm{~m}{ }^{2}$ \\
\hline Wheel radius & $R_{e}$ & 0.3 & $\mathrm{~m}$ \\
\hline Time constant of braking system & $\gamma_{T}$ & 0.1 & $\mathrm{~s}$ \\
\hline Wheel moment of inertia & $I_{w}$ & 2 & $\mathrm{~kg} \mathrm{~m}^{2}$ \\
\hline Relaxation length & $\sigma$ & 0.3 & $\mathrm{~m}$ \\
\hline Aerodynamic coefficient & $K_{D}$ & 0.36 & $\mathrm{~kg} / \mathrm{m}$ \\
\hline Tire-force parameters & Notation & $\begin{array}{c}\text { Value } \\
i \in\{1,2\}\end{array}$ & $\begin{array}{c}\text { Value } \\
i \in\{3,4\}\end{array}$ \\
\hline \multirow[t]{7}{*}{ Longitudinal force } & $\mu_{x, i}$ & 1.1959 & 1.2027 \\
\hline & $B_{x, i}$ & 11.6848 & 11.1217 \\
\hline & $C_{x, i}$ & 1.685 & 1.685 \\
\hline & $E_{x, i}$ & 0.37729 & 0.36192 \\
\hline & $B_{x 1, i}$ & 12.35 & 12.35 \\
\hline & $B_{x 2, i}$ & -10.77 & -10.77 \\
\hline & $C_{x \alpha, i}$ & 1.092 & 1.092 \\
\hline \multirow[t]{7}{*}{ Lateral force } & $\mu_{y, i}$ & 0.93476 & 0.96146 \\
\hline & $B_{y, i}$ & 8.8626 & 9.3016 \\
\hline & $C_{y, i}$ & 1.193 & 1.193 \\
\hline & $E_{y, i}$ & -1.2076 & -1.1087 \\
\hline & $B_{y 1, i}$ & 6.461 & 6.461 \\
\hline & $B_{y 2, i}$ & 4.196 & 4.196 \\
\hline & $C_{y \kappa, i}$ & 1.081 & 1.081 \\
\hline
\end{tabular}


Table A.2: Vehicle model parameters used in Papers IV and V. The model parameters are chosen to resemble the Ford Focus like vehicle model available in CarMaker [25].

\begin{tabular}{|c|c|c|c|}
\hline Description & Notation & Value & Unit \\
\hline \multirow{2}{*}{ Longitudinal distance to wheel $i$} & $l_{x, 1}, l_{x, 2}$ & 1.043 & $\mathrm{~m}$ \\
\hline & $l_{x, 3}, l_{x, 4}$ & -1.637 & $\mathrm{~m}$ \\
\hline \multirow{2}{*}{ Lateral distance to wheel $i$} & $l_{y, 1}, l_{y, 3}$ & 0.755 & $\mathrm{~m}$ \\
\hline & $l_{y, 2}, l_{y, 4}$ & -0.755 & $\mathrm{~m}$ \\
\hline Height of the center of mass & $h$ & 0.305 & $\mathrm{~m}$ \\
\hline Vehicle mass & $m$ & 1174 & $\mathrm{~kg}$ \\
\hline Roll moment of inertia & $I_{x x}$ & 441 & $\mathrm{~kg} \mathrm{~m}^{2}$ \\
\hline Pitch moment of inertia & $I_{y y}$ & 1569 & $\mathrm{~kg} \mathrm{~m}^{2}$ \\
\hline Yaw moment of inertia & $I_{z z}$ & 1730 & $\mathrm{~kg} \mathrm{~m}^{2}$ \\
\hline Pitch suspension stiffness & $K_{\theta}$ & 363540 & $\mathrm{Nm} / \mathrm{rad}$ \\
\hline Pitch suspension damping & $D_{\theta}$ & 30960 & $\mathrm{Nms} / \mathrm{rad}$ \\
\hline Roll suspension stiffness & $K_{\phi}$ & 178000 & $\mathrm{Nm} / \mathrm{rad}$ \\
\hline Roll suspension damping & $D_{\phi}$ & 16000 & $\mathrm{Nms} / \mathrm{rad}$ \\
\hline Rotational wheel inertia & $I_{w}$ & 1 & $\mathrm{~kg} \mathrm{~m}^{2}$ \\
\hline Wheel radius & $R_{e}$ & 0.293 & m \\
\hline Time constant of braking system & $\gamma_{T}$ & 0.1 & $\mathrm{~s}$ \\
\hline Wheel moment of inertia & $I_{w}$ & 1 & $\mathrm{~kg} \mathrm{~m}^{2}$ \\
\hline Relaxation length & $\sigma$ & 0.1 & $\mathrm{~m}$ \\
\hline Aerodynamic coefficient & $K_{D}$ & 0.36 & $\mathrm{~kg} / \mathrm{m}$ \\
\hline Tire-force parameters & Notation & $\begin{array}{c}\text { Value } \\
i \in\{1,2\}\end{array}$ & $\begin{array}{c}\text { Value } \\
i \in\{3,4\}\end{array}$ \\
\hline \multirow[t]{7}{*}{ Longitudinal force } & $\mu_{x, i}$ & 1.0530 & 1.1016 \\
\hline & $B_{x, i}$ & 19.6942 & 17.7938 \\
\hline & $C_{x, i}$ & 1.4739 & 1.5161 \\
\hline & $E_{x, i}$ & -0.2210 & -0.1656 \\
\hline & $B_{x 1, i}$ & 15.3728 & 15.3728 \\
\hline & $B_{x 2, i}$ & -8.7422 & -8.7422 \\
\hline & $C_{x \alpha, i}$ & 1.0544 & 1.0544 \\
\hline \multirow[t]{7}{*}{ Lateral force } & $\mu_{y, i}$ & 1.0386 & 1.0961 \\
\hline & $B_{y, i}$ & 9.3917 & 7.0765 \\
\hline & $C_{y, i}$ & 1.5814 & 1.9103 \\
\hline & $E_{y, i}$ & -0.0569 & 0.3342 \\
\hline & $B_{y 1, i}$ & 4.5491 & 4.5491 \\
\hline & $B_{y 2, i}$ & 8.3054 & 8.3054 \\
\hline & $C_{u \kappa, i}$ & 1.9619 & 1.9619 \\
\hline
\end{tabular}





\section{Appendix B}

\section{The Modified Hamiltonian Algorithm}

7 He Modified Hamiltonian Algorithm (MHA) by Gao, Lidberg, and 1 Gordon [77] is used in Paper V, and is for completeness described in detail here. A significant aspect of the method is also used in Papers IIIIV.

In a first step, MHA uses a friction-limited particle model to determine a center-of-mass acceleration-vector reference $a^{\mathrm{d}}$. Assuming that this reference is at the limits of friction, the reference is achieved by minimizing the function

$$
H=p_{x} F_{x}+p_{y} F_{y}, \quad\left[\begin{array}{c}
p_{x} \\
p_{y}
\end{array}\right]=-\frac{a^{\mathrm{d}}}{\left|a^{\mathrm{d}}\right|},
$$

where $F_{x}$ and $F_{y}$ are the longitudinal and lateral forces acting on the vehicle, respectively. To include control of the yaw moment $M_{z}$, the Hamiltonian function $H$ is adapted to

$$
H=p_{x} F_{x}+p_{y} F_{y}+\lambda M_{z},
$$

where $\lambda$ acts similar to a Lagrange multiplier and constrains the yaw moment $M_{z}$ to a desired yaw moment $M_{z}^{\mathrm{d}}$. This is achieved by adapting 
the value of $\lambda$ online by a fixed increment $\Delta$ at each controller sampling instant using

$$
\lambda \rightarrow \lambda+\Delta \cdot \operatorname{sign}\left(M_{z}-M_{z}^{\mathrm{d}}\right) .
$$

Expressing the individual tire forces $\left[F_{x, i}^{v}, F_{y, i}^{v}\right]^{\mathrm{T}}, \forall i \in\{1,2,3,4\}$, in vehicle coordinates, the total forces and moment are given as

$$
\begin{aligned}
F_{x} & =\sum_{i=1}^{4} F_{x, i}^{v}, \\
F_{y} & =\sum_{i=1}^{4} F_{y, i}^{v}, \\
M_{z} & =\sum_{i=1}^{4}\left(l_{x, i} F_{y, i}^{v}-l_{y, i} F_{x, i}^{v}\right) .
\end{aligned}
$$

The modified Hamiltonian function (B.2) can then be expressed as

$$
\begin{aligned}
H & =\sum_{i=1}^{4} p_{x} F_{x, i}^{v}+p_{y} F_{y, i}^{v}+\lambda\left(l_{x, i} F_{y, i}^{v}-l_{y, i} F_{x, i}^{v}\right) \\
& =\sum_{i=1}^{4}\left(p_{x}-\lambda l_{y, i}\right) F_{x, i}^{v}+\left(p_{y}+\lambda l_{x, i}\right) F_{y, i}^{v} \\
& =\sum_{i=1}^{4} p_{x, i}^{v} F_{x, i}^{v}+p_{y, i}^{v} F_{y, i}^{v} .
\end{aligned}
$$

This can be written as a sum of local Hamiltonian functions $H=\sum_{i=1}^{4} H_{i}$ and can be converted to local tire forces by using

$$
\left[\begin{array}{c}
F_{x, i}^{v} \\
F_{y, i}^{v}
\end{array}\right]=\left[\begin{array}{cc}
\cos \left(\delta_{i}\right) & -\sin \left(\delta_{i}\right) \\
\sin \left(\delta_{i}\right) & \cos \left(\delta_{i}\right)
\end{array}\right]\left[\begin{array}{c}
F_{x, i} \\
F_{y, i}
\end{array}\right] .
$$

The procedure described so far was also adopted in Papers III-IV, but those papers used different procedures to minimize the function (B.2) than what was used in MHA [77]. What follows here is the procedure to minimize (B.2) introduced in [77] and used in Paper V.

At each wheel, a one-dimensional search is carried out to find the desired slip ratio $\kappa_{i}^{\mathrm{d}}$ that minimizes the local Hamiltonian function $H_{i}$ for the given slip angle $\alpha_{i}$,

$$
\kappa_{i}^{\mathrm{d}}=\underset{\kappa_{i}}{\arg \min } H_{i} .
$$


This results in the predicted tire forces

$$
\begin{aligned}
& F_{x, i}^{\mathrm{p}}=F_{x, i}\left(\kappa_{i}^{\mathrm{d}}, \alpha_{i}\right), \\
& F_{y, i}^{\mathrm{p}}=F_{y, i}\left(\kappa_{i}^{\mathrm{d}}, \alpha_{i}\right) .
\end{aligned}
$$

Assuming steady-state wheel dynamics, the corresponding braking torque $T_{i}$ is computed as

$$
T_{i}=R_{e} F_{x, i}^{\mathrm{p}} .
$$

where $R_{e}$ is the effective wheel radius.

To determine the desired body slip and steering angle, local minimizations of the Hamiltonian (B.2) are performed. Via small perturbations $\pm \varepsilon$ of the slip angle $\alpha_{i}$ at the desired slip ratio $\kappa_{i}^{\mathrm{d}}$, it is approximated that

$$
\frac{\partial H_{i}}{\partial \alpha_{i}} \approx \frac{H_{i}\left(\alpha_{i}+\varepsilon\right)-H_{i}\left(\alpha_{i}-\varepsilon\right)}{2 \varepsilon} .
$$

Further, the slip angle $\alpha_{i}$ for small slip angles $\beta$ can be approximated by

$$
\alpha_{i} \approx \delta_{i}+\beta-\frac{l_{x, i} \dot{\psi}}{v_{x}} .
$$

Considering a typical vehicle with front-wheel steering $\left(\delta_{1}=\delta_{2}=\delta\right.$ and $\left.\delta_{3}=\delta_{4}=0\right)$, the influence of a change of steering angle $\delta$ and body slip $\beta$ on the value of (B.2) are using the chain rule and (B.14) given by

$$
\begin{aligned}
& \frac{\partial H}{\partial \delta}=\frac{\partial H_{1}}{\partial \alpha_{1}} \frac{\partial \alpha_{1}}{\partial \delta}+\frac{\partial H_{2}}{\partial \alpha_{2}} \frac{\partial \alpha_{2}}{\partial \delta} \approx \frac{\partial H_{1}}{\partial \alpha_{1}}+\frac{\partial H_{2}}{\partial \alpha_{2}}, \\
& \frac{\partial H}{\partial \beta}=\sum_{i=1}^{4} \frac{\partial H_{i}}{\partial \alpha_{i}} \cdot \frac{\partial \alpha_{i}}{\partial \beta} \approx \sum_{i=1}^{4} \frac{\partial H_{i}}{\partial \alpha_{i}} .
\end{aligned}
$$

This is now used to create control laws that reduce the value of (B.2). The common front-steering rate $\dot{\delta}$ acts to locally reduce the value of (B.2) via

$$
\dot{\delta}= \begin{cases}-\dot{\delta}_{\max } \operatorname{sign}\left(\frac{\partial H}{\partial \delta}\right) & \text { if }\left|\frac{\partial H}{\partial \delta}\right|>\text { tolerance } \\ 0 & \text { otherwise }\end{cases}
$$

where $\dot{\delta}_{\text {max }}$ is a parameter determining the steering-rate limit. Similarly, the target body-slip rate $\dot{\beta}^{H}$ acts to locally reduce the value of (B.2) via

$$
\dot{\beta}^{H}= \begin{cases}-\dot{\beta}_{\max } \operatorname{sign}\left(\frac{\partial H}{\partial \beta}\right) & \text { if }\left|\frac{\partial H}{\partial \beta}\right|>\text { tolerance } \\ 0 & \text { otherwise }\end{cases}
$$


where $\dot{\beta}_{\max }$ is a parameter determining the body-slip rate limit. To prevent large body slip $\beta$, thresholds $\beta_{1}$ and $\beta_{2}$ are introduced such that $0<\beta_{1}<\beta_{2}$ and the desired body-slip rate $\dot{\beta}^{\mathrm{d}}$ is determined by

$$
\dot{\beta}^{\mathrm{d}}= \begin{cases}-\dot{\beta}_{\max } \operatorname{sign}(\beta) & \text { if }|\beta|>\beta_{2}, \\ 0 & \text { if }|\beta|>\beta_{1} \text { and } \beta \dot{\beta}_{H}>0, \\ \dot{\beta}^{H} & \text { otherwise. }\end{cases}
$$

To complete the control loop, the desired yaw moment $M_{z}^{\mathrm{d}}$ in (B.3) is determined. The desired yaw rate $\dot{\psi}^{\mathrm{d}}$ is computed as

$$
\dot{\psi}^{\mathrm{d}}=\dot{\phi}+\dot{\beta}^{\mathrm{d}},
$$

where $\dot{\phi}$ is related to the vehicle path curvature $\rho$ and velocity $v$ by $\dot{\phi}=v \rho$. Computing the predicted path-lateral force $F_{\perp}^{\mathrm{p}}$ as

$$
\begin{aligned}
F_{\perp}^{\mathrm{p}} & =F_{y}^{\mathrm{p}} \cos (\beta)-F_{x}^{\mathrm{p}} \sin (\beta), \\
{\left[\begin{array}{c}
F_{x}^{\mathrm{p}} \\
F_{y}^{\mathrm{p}}
\end{array}\right] } & =\sum_{i=1}^{4}\left[\begin{array}{cc}
\cos \left(\delta_{i}\right) & -\sin \left(\delta_{i}\right) \\
\sin \left(\delta_{i}\right) & \cos \left(\delta_{i}\right)
\end{array}\right]\left[\begin{array}{c}
F_{x, i}^{\mathrm{p}} \\
F_{y, i}^{\mathrm{p}}
\end{array}\right],
\end{aligned}
$$

the current vehicle path curvature $\rho$ is approximated as

$$
\rho=\frac{F_{\perp}^{\mathrm{p}}}{m v^{2}},
$$

where $m$ is the vehicle mass. Finally, the loop is closed around the desired yaw rate $\dot{\psi}^{\text {d }}$ by computing $M_{z}^{\mathrm{d}}$ using a first-order control law with characterizing time constant $\tau$ :

$$
M_{z}^{\mathrm{d}}=\frac{I_{z z}\left(\dot{\psi}^{\mathrm{d}}-\dot{\psi}\right)}{\tau},
$$

where $I_{z z}$ is the yaw moment of inertia. 


\section{References}

[1] Transportstyrelsen, "Dödade, skadade, bilar i trafik, bensinleveranser och invånare samt dödade per 100000 bilar respektive invånare (årsvis fr.o.m. 1950)," 2020, in Swedish. Accessed: 2020-10-11. URL: https://www.transportstyrelsen.se/globalassets/global/press/statistik/ olycksstatistik/p_nationell_befolkning_bilar_bensin_1950-.xlsx

[2] U. Brüde, "Sveriges trafiksäkerhet i ett 100-årigt perspektiv," 2012, in Swedish.

[3] C. Shelden, "Prevention, the only cure for head injuries resulting from automobile accidents," Journal of the American Medical Association, vol. 159, no. 10, pp. 981-986, 1955.

[4] U. Kiencke and L. Nielsen, Automotive control systems: for engine, driveline and vehicle., 2nd ed. Berlin, Germany: Springer-Verlag, 2005.

[5] M. Rizzi, J. Strandroth, A. Kullgren, C. Tingvall, and B. Fildes, "Effectiveness of motorcycle antilock braking systems (ABS) in reducing crashes, the first cross-national study," Traffic Injury Prevention, vol. 16, no. 2, pp. $177-183,2015$.

[6] P. Cummings and D. C. Grossman, "Antilock brakes and the risk of driver injury in a crash: A case-control study," Accident Analysis $\&$ Prevention, vol. 39, no. 5, pp. 995-1000, 2007.

[7] C. J. Kahane and J. N. Dang, "The long-term effect of ABS in passenger cars and LTVs," Evaluation Division, National Center for Statistics and Analysis, National Highway Traffic Safety Administration, U.S. Department of Transportation, NHTSA Technical Report DOT HS 811 182, 2009.

[8] A. Lyckegaard, T. Hels, and I. M. Bernhoft, "Effectiveness of electronic stability control on single-vehicle accidents," Traffic Injury Prevention, vol. 16, no. 4, pp. 380-386, 2015.

[9] G. Genta and L. Morello, The Automotive Chassis, Mechanical Engineering Series. New York, NY, United States: Springer-Verlag, 2008. 
[10] R. Rajamani, Vehicle Dynamics and Control, 2nd ed. New York, NY, United States: Springer-Verlag, 2012.

[11] T. Gordon and M. Lidberg, "Automated driving and autonomous functions on road vehicles," Vehicle System Dynamics, vol. 53, no. 7, pp. 958-994, 2015 .

[12] J. B. Cicchino, "Effectiveness of forward collision warning and autonomous emergency braking systems in reducing front-to-rear crash rates," Accident Analysis \& Prevention, vol. 99, pp. 142-152, 2017.

[13] Euro NCAP, "2020 assisted driving tests," 2020, Accessed: 2020-1011. URL: https://www.euroncap.com/en/vehicle-safety/safety-campaigns/ 2020-assisted-driving-tests

[14] National Transportation Safety Board (NTSB), "Collision between a sport utility vehicle operating withpartial driving automation and a crash attenuator, Mountain View, California, March 23, 2018," Washington, DC, United States, NTSB Highway Accident Report NTSB/HAR-20/01, 2020.

[15] National Transportation Safety Board (NTSB), "Collision between car operating with partial driving automation and truck-tractor semitrailer, Delray Beach, Florida, March 1, 2019," Washington, DC, United States, NTSB Highway Accident Brief NTSB/HAB-20/01, 2020.

[16] SAE International, "Taxonomy and definitions for terms related to driving automation systems for on-road motor vehicles. Standard J3016_201806," 2018.

[17] ERTRAC Working Group, Connectivity and Automated Driving, "Connected automated driving roadmap," Brussel, Belgium, Technical Report, 2019, Accessed: 2020-10-11. URL: https://www.ertrac.org/uploads/ documentsearch/id57/ERTRAC-CAD-Roadmap-2019.pdf

[18] National Highway Traffic Safety Administration, U.S. Department of Transportation (NHTSA), "Federal automated vehicles policy," 2016.

[19] R. Spekking, "Mercedes AMG CLS 55 - Demonstration of drifting at the "Fahrsicherheitszentrum Nürburgring"," 2006, Accessed: 2020-10-11. URL: https://commons.wikimedia.org/wiki/File: Mercedes_AMG_CLS_55___Demonstration_of_drifting_1b.jpg

[20] R. S. Sharp and H. Peng, "Vehicle dynamics applications of optimal control theory," Vehicle System Dynamics, vol. 49, no. 7, pp. 1073-1111, 2011.

[21] H. Waschl, I. Kolmanovsky, M. Steinbuch, and L. del Re, Optimization and Optimal Control in Automotive Systems, Lecture Notes in Control and Information Sciences. Berlin, Germany: Springer-Verlag, 2014, vol. 455. 
[22] B. Olofsson, "Topics in machining with industrial robot manipulators and optimal motion control," Ph.D. dissertation, No. TFRT-1108, Department of Automatic Control, Lund University, Sweden, 2015.

[23] P. Anistratov, "Computation of autonomous safety maneuvers using segmentation and optimization," Licentiate Thesis, Linköping Studies in Science and Technology. No. 1860, Department of Electrical Engineering, Linköping University, Sweden, 2019.

[24] V. Fors, "Optimal braking patterns and forces in autonomous safety-critical maneuvers," Licentiate Thesis, Linköping Studies in Science and Technology. No. 1804, Department of Electrical Engineering, Linköping University, Sweden, 2018.

[25] IPG Automotive GmbH, "Carmaker: Virtual testing of automobiles and light-duty vehicles," 2019. URL: https://ipg-automotive.com/productsservices/simulation-software/carmaker

[26] R. Oliveira, "Motion planning for heavy-duty vehicles," Licentiate Thesis, TRITA-EECS-AVL. No. 2019:56, Division of Decision and Control Systems, KTH Royal Institute of Technology, Sweden, 2019.

[27] Passenger cars - Test track for a severe lane-change manoeuvre — Part 2: Obstacle avoidance, ISO Standard 3888-2:2011, 2011.

[28] K. Bergman, "On motion planning using numerical optimal control," Licentiate Thesis, Linköping Studies in Science and Technology. No. 1843, Department of Electrical Engineering, Linköping University, Sweden, 2019.

[29] Y. Kuwata, J. Teo, G. Fiore, S. Karaman, E. Frazzoli, and J. P. How, "Realtime motion planning with applications to autonomous urban driving," IEEE Transactions on Control Systems Technology, vol. 17, no. 5, pp. 11051118, 2009.

[30] M. Brown and J. C. Gerdes, "Coordinating tire forces to avoid obstacles using nonlinear model predictive control," IEEE Transactions on Intelligent Vehicles, vol. 5, no. 1, pp. 21-31, 2020.

[31] E. N. Smith, E. Velenis, D. Tavernini, and D. Cao, "Effect of handling characteristics on minimum time cornering with torque vectoring," Vehicle System Dynamics, vol. 56, no. 2, pp. 221-248, 2018.

[32] N. R. Kapania and J. C. Gerdes, "Design of a feedback-feedforward steering controller for accurate path tracking and stability at the limits of handling," Vehicle System Dynamics, vol. 53, no. 12, pp. 1687-1704, 2015.

[33] J. K. Subosits and J. C. Gerdes, "From the racetrack to the road: Realtime trajectory replanning for autonomous driving," IEEE Transactions on Intelligent Vehicles, vol. 4, no. 2, pp. 309-320, 2019. 
[34] Z. Shiller and S. Sundar, "Emergency lane-change maneuvers of autonomous vehicles," ASME Journal of Dynamic Systems, Measurement and Control, vol. 120 , no. 1 , pp. 37-44, 1998.

[35] M. Klomp, M. Lidberg, and T. J. Gordon, "On optimal recovery from terminal understeer," Proceedings of the Institution of Mechanical Engineers, Part D: Journal of Automobile Engineering, vol. 228, no. 4, pp. 412-425, 2014 .

[36] S. C. Peters, "Optimal planning and control for hazard avoidance of frontwheel steered ground vehicles," Ph.D. dissertation, Department of Mechanical Engineering, Massachusetts Institute of Technology, 2012.

[37] Y. Gao, T. Gordon, D. Yang, M. Jonasson, and B. Jacobsson, "Robust automated collision avoidance using an updating particle reference," presented at the 14th International Symposium on Advanced Vehicle Control (AVEC), Beijing, China, 2018.

[38] A. S. P. Singh and O. Nishihara, "Nondimensionalized univariate equation characterizing optimal state feedback control for collision avoidance," IEEE Transactions on Intelligent Transportation Systems, vol. 19, no. 10, pp. 3344$3359,2018$.

[39] J. Wong, Theory of Ground Vehicles, 4th ed. Hoboken, NJ, United States: John Wiley \& Sons, Inc., 2008.

[40] T. Fraichard and C. Laugier, "Path-velocity decomposition revisited and applied to dynamic trajectory planning," in Proceedings of the 1993 IEEE International Conference on Robotics and Automation (ICRA), pp. 40-45 vol.2, Atlanta, GA, United States, 1993.

[41] M. Morsali, E. Frisk, and J. Åslund, "Real-time velocity planning for heavy duty truck with obstacle avoidance," in Proceedings of the 2017 IEEE Intelligent Vehicles Symposium (IV), pp. 109-114, Los Angeles, CA, United States, 2017.

[42] O. Ljungqvist, "Motion planning and feedback control techniques with applications to long tractor-trailer vehicles," Ph.D. dissertation, Linköping Studies in Science and Technology. No. 2070, Department of Electrical Engineering, Linköping University, Sweden, 2020.

[43] B. Paden, M. Čáp, S. Z. Yong, D. Yershov, and E. Frazzoli, "A survey of motion planning and control techniques for self-driving urban vehicles," IEEE Transactions on Intelligent Vehicles, vol. 1, no. 1, pp. 33-55, 2016.

[44] G. C. Pereira, "Lateral model predictive control for autonomous heavy-duty vehicles : Sensor, actuator, and reference uncertainties," Licentiate Thesis, TRITA-EECS-AVL. No. 2020:38, Division of Decision and Control Systems, KTH Royal Institute of Technology, Sweden, 2020. 
[45] H. Goldstein, C. Poole, and J. Safko, Classical Mechanics, 3rd ed. London, United Kingdom: Pearson, 2013.

[46] K. Lundahl, J. Åslund, and L. Nielsen, "Vehicle dynamics platform, experiments, and modeling aiming at critical maneuver handling," Department of Electrical Engineering, Linköping University, Sweden, Technical Report 3064, 2013.

[47] C. Hatipoglu, U. Ozguner, and K. A. Redmill, "Automated lane change controller design," IEEE Transactions on Intelligent Transportation Systems, vol. 4, no. 1, pp. 13-22, 2003.

[48] K. Kritayakirana and J. C. Gerdes, "Autonomous vehicle control at the limits of handling," International Journal of Vehicle Autonomous Systems, vol. 10, no. 4, pp. 271-296, 2012.

[49] H. Zhang and J. Wang, "Vehicle lateral dynamics control through AFS/DYC and robust gain-scheduling approach," IEEE Transactions on Vehicular Technology, vol. 65, no. 1, pp. 489-494, 2016.

[50] S. Lee and C. C. Chung, "Predictive control with sliding mode for autonomous driving vehicle lateral maneuvering," in Proceedings of the 2017 American Control Conference (ACC), pp. 2998-3003, Seattle, WA, United States, 2017.

[51] M. Brown, J. Funke, S. Erlien, and J. C. Gerdes, "Safe driving envelopes for path tracking in autonomous vehicles," Control Engineering Practice, vol. 61, pp. 307-316, 2017.

[52] M. Veneri and M. Massaro, "The effect of Ackermann steering on the performance of race cars," Vehicle System Dynamics, 2020, DOI 10.1080/00423114.2020.1730917.

[53] M. Jonasson, J. Andreasson, B. Jacobson, and A. Stensson Trigell, "Global force potential of over-actuated vehicles," Vehicle System Dynamics, vol. 48, no. 9, pp. 983-998, 2010.

[54] K. Lundahl, B. Olofsson, K. Berntorp, J. Åslund, and L. Nielsen, "Towards lane-keeping electronic stability control for road-vehicles," in Proceedings of the 19th IFAC World Congress, vol. 47, no. 3, pp. 6319-6325, Cape Town, South Africa, 2014.

[55] D. Yang, T. J. Gordon, B. Jacobson, and M. Jonasson, "Quasi-linear optimal path controller applied to post impact vehicle dynamics," IEEE Transactions on Intelligent Transportation Systems, vol. 13, no. 4, pp. 1586-1598, 2012. 
[56] K. Berntorp, "Derivation of a six degrees-of-freedom ground-vehicle model for automotive applications," Department of Automatic Control, Lund University, Sweden, Technical Report 7627, 2013.

[57] S. Uhlar, F. Heyder, and T. König, "Assessment of two physical tyre models in relation to their NVH performance up to $300 \mathrm{~Hz}$," Vehicle System Dynamics, 2019, DOI 10.1080/00423114.2019.1681475.

[58] H. B. Pacejka, Tyre and Vehicle Dynamics, 2nd ed. Oxford, United Kingdom: Butterworth-Heinemann, 2006.

[59] J. Svendenius, "Tire modeling and friction estimation," Ph.D. dissertation, No. TFRT-1077, Department of Automatic Control, Lund University, Sweden, 2007.

[60] H. Mouri and H. Furusho, "Automatic path tracking using linear quadratic control theory," in Proceedings of the 1997 IEEE Intelligent Transportation Systems Conference (ITSC), pp. 948-953, Boston, MA, United States, 1997.

[61] A. Gupta, M. Nilsson, P. Falcone, E. Klintberg, and L. J. Mårdh, “A framework for vehicle lateral motion control with guaranteed tracking and performance," in Proceedings of the 2019 IEEE Intelligent Transportation Systems Conference (ITSC), pp. 3607-3612, Auckland, New Zealand, 2019.

[62] F. Fiala, "Seitenkrafte am rollenden luftreifen," VDI Zeitschrift, vol. 96, no. 29, pp. 973-979, 1954, in German.

[63] V. A. Laurense and J. C. Gerdes, "Speed control for robust path-tracking for automated vehicles at the tire-road friction limit," presented at the 14th International Symposium on Advanced Vehicle Control (AVEC), Beijing, China, 2018.

[64] C. E. Beal, "Rapid road friction estimation using independent left/right steering torque measurements," Vehicle System Dynamics, vol. 58, no. 3, pp. 377-403, 2020.

[65] A. O'Neill, P. Gruber, J. F. Watts, and J. Prins, "Predicting tyre behaviour on different road surfaces," in Proceedings of the 26th Symposium of the International Association of Vehicle System Dynamics (IAVSD), pp. 18991908, Gothenburg, Sweden, 2019.

[66] L. Romano, F. Bruzelius, and B. Jacobson, "Unsteady-state brush theory," Vehicle System Dynamics, 2020, DOI 10.1080/00423114.2020.1774625.

[67] F. Braghin, F. Cheli, and E. Sabbioni, "Environmental effects on Pacejka's scaling factors," Vehicle System Dynamics, vol. 44, no. 7, pp. 547-568, 2006. 
[68] B. Olofsson, K. Lundahl, K. Berntorp, and L. Nielsen, "An investigation of optimal vehicle maneuvers for different road conditions," in Proceedings of the rth IFAC Symposium on Advances in Automotive Control (AAC), vol. 46, no. 21, pp. 66-71, Tokyo, Japan, 2013.

[69] R. Zhang, Z. He, H. Wang, F. You, and K. Li, "Study on self-tuning tyre friction control for developing main-servo loop integrated chassis control system," IEEE Access, vol. 5, pp. 6649-6660, 2017.

[70] K. Berntorp, R. Quirynen, T. Uno, and S. D. Cairano, "Trajectory tracking for autonomous vehicles on varying road surfaces by friction-adaptive nonlinear model predictive control," Vehicle System Dynamics, vol. 58, no. 5, pp. 705-725, 2020.

[71] D. Yang, B. Jacobson, M. Jonasson, and T. J. Gordon, "Closed-loop controller for post-impact vehicle dynamics using individual wheel braking and front axle steering," International Journal of Vehicle Autonomous Systems, vol. 12, no. 2, pp. 158-179, 2014.

[72] J. Y. Goh, T. Goel, and J. C. Gerdes, "Toward automated vehicle control beyond the stability limits: Drifting along a general path," ASME Journal of Dynamic Systems, Measurement, and Control, vol. 142, no. 2, 112019.

[73] K. Berntorp, B. Olofsson, K. Lundahl, and L. Nielsen, "Models and methodology for optimal trajectory generation in safety-critical road-vehicle manoeuvres," Vehicle System Dynamics, vol. 52, no. 10, pp. 1304-1332, 2014.

[74] V. Fors, B. Olofsson, and L. Nielsen, "Formulation and interpretation of optimal braking and steering patterns towards autonomous safety-critical manoeuvres," Vehicle System Dynamics, vol. 57, no. 8, pp. 1206-1223, 2019.

[75] V. Fors, B. Olofsson, and L. Nielsen, "Attainable force volumes of optimal autonomous at-the-limit vehicle manoeuvres," Vehicle System Dynamics, vol. 58, no. 7, pp. 1101-1122, 2020.

[76] V. Fors, B. Olofsson, and L. Nielsen, "Yaw-moment control at-the-limit of friction using individual front-wheel steering and four-wheel braking," in Proceedings of the 9th IFAC International Symposium on Advances in Automotive Control (AAC), vol. 52, no. 5, pp. 458-464, Orléans, France, 2019.

[77] Y. Gao, M. Lidberg, and T. J. Gordon, "Modified Hamiltonian algorithm for optimal lane change with application to collision avoidance." MM Science Journal, no. MAR 2015, pp. 576-584, 2015.

[78] V. Fors, B. Olofsson, and L. Nielsen, "Autonomous wary collision avoidance," IEEE Transactions on Intelligent Vehicles, 2020, DOI 10.1109/TIV.2020.3029853. 
[79] V. Fors, Y. Gao, B. Olofsson, T. J. Gordon, and L. Nielsen, "Real-time minimum-time lane change using the modified Hamiltonian algorithm," in Proceedings of the 26th Symposium of the International Association of Vehicle System Dynamics (IAVSD), pp. 1457-1465, Gothenburg, Sweden, 2019 .

[80] V. Fors, P. Anistratov, B. Olofsson, and L. Nielsen, "Predictive force-centric emergency collision avoidance by steering and individual four-wheel braking," 2020, Manuscript.

[81] V. Fors, B. Olofsson, and L. Nielsen, "Formulation and interpretation of optimal braking patterns in autonomous lane-keeping maneuvers," presented at the 2nd IAVSD Workshop on Dynamics of Road Vehicles, Berlin, Germany, 2017.

[82] V. Fors, B. Olofsson, and L. Nielsen, "Slip-angle feedback control for autonomous safety-critical maneuvers at-the-limit of friction," presented at the 14th International Symposium on Advanced Vehicle Control (AVEC), Beijing, China, 2018. URL: http://urn.kb.se/resolve?urn=urn:nbn:se:liu: diva-149899

[83] B. Olofsson and L. Nielsen, "Using crash databases to predict effectiveness of new autonomous vehicle maneuvers for lane-departure injury reduction," IEEE Transactions on Intelligent Transportation Systems, 2020, DOI 10.1109/TITS.2020.2983553.

[84] V. A. Laurense, J. Y. Goh, and J. C. Gerdes, "Path-tracking for autonomous vehicles at the limit of friction," in Proceedings of the 2017 American Control Conference (ACC), pp. 5586-5591, Seattle, WA, United States, 2017.

[85] M. Ali, P. Falcone, C. Olsson, and J. Sjöberg, "Predictive prevention of loss of vehicle control for roadway departure avoidance," IEEE Transactions on Intelligent Transportation Systems, vol. 14, pp. 56-68, 2013.

[86] L. Svensson, M. Bujarbaruah, A. Karsolia, C. Berger, and M. Törngren, "Traction adaptive motion planning at the limits of handling," 2020, arXiv:2009.04180 [cs.RO]. URL: https://arxiv.org/abs/2009.04180

[87] K. Lundahl, "Models and critical maneuvers for road vehicles," Ph.D. dissertation, Linköping Studies in Science and Technology. No. 1763, Department of Electrical Engineering, Linköping University, Sweden, 2016. 
Papers 


\section{Papers}

The papers associated with this thesis have been removed for copyright reasons. For more details about these see:

http://urn.kb.se/resolve?urn=urn:nbn:se:liu:diva-170606 


\section{FACULTY OF SCIENCE AND ENGINEERING}

Linköping Studies in Science and Technology

Dissertations No. 2102, 2020

Department of Electrical Engineering

Linköping University

SE-581 83 Linköping, Sweden

www.liu.se 